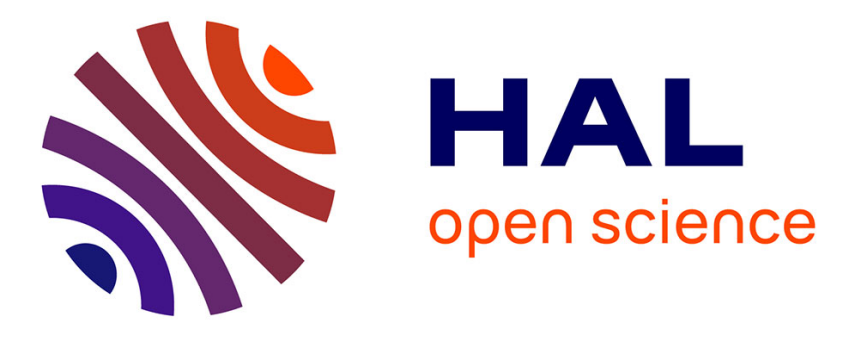

\title{
A cooperative conjugate gradient method for linear systems permitting efficient multi-thread implementation
}

Amit A Bhaya, Pierre-Alexandre A Bliman, Guilherme Niedu, Fernando A Pazos

\section{To cite this version:}

Amit A Bhaya, Pierre-Alexandre A Bliman, Guilherme Niedu, Fernando A Pazos. A cooperative conjugate gradient method for linear systems permitting efficient multi-thread implementation. Computational \& Applied Mathematics, 2017, pp.1-28. 10.1007/s40314-016-0416-7 . hal-01558765

\section{HAL Id: hal-01558765 \\ https://hal.inria.fr/hal-01558765}

Submitted on 10 Jul 2017

HAL is a multi-disciplinary open access archive for the deposit and dissemination of scientific research documents, whether they are published or not. The documents may come from teaching and research institutions in France or abroad, or from public or private research centers.
L'archive ouverte pluridisciplinaire HAL, est destinée au dépôt et à la diffusion de documents scientifiques de niveau recherche, publiés ou non, émanant des établissements d'enseignement et de recherche français ou étrangers, des laboratoires publics ou privés. 


\title{
A cooperative conjugate gradient method for linear systems permitting efficient multi-thread implementation
}

\author{
Amit Bhaya · Pierre-Alexandre Bliman • \\ Guilherme Niedu • Fernando A. Pazos
}

Received: date / Accepted: date

\begin{abstract}
This paper revisits, in a multi-thread context, the so-called multiparameter or block conjugate gradient (BCG) methods, first proposed as sequential algorithms by O'Leary and Brezinski, for the solution of the linear system $\mathbf{A x}=\mathbf{b}$, for an $n$-dimensional symmetric positive definite matrix $\mathbf{A}$. Instead of the scalar parameters of the classical CG algorithm, which minimizes a scalar functional at each iteration, multiple descent and conjugate directions are updated simultaneously. Implementation involves the use of multiple threads and the algorithm is referred to as cooperative CG (CCG) in order to emphasize that each thread now uses information that comes from the other threads. It is shown that for a sufficiently large matrix dimension $n$, the use of an optimal number of threads results in a worst case flop count of $O\left(n^{7 / 3}\right)$ in exact arithmetic. Numerical experiments on a multicore, multi-thread computer, for synthetic and real matrices, illustrate the theoretical results.
\end{abstract}

Keywords Discrete linear systems; Iterative methods; Conjugate gradient algorithm; Cooperative algorithms;

Mathematics Subject Classification (2000) 65Y05

Bhaya

Department of Electrical Engineering, Federal University of Rio de Janeiro, Rio de Janeiro - RJ, Brazil. E-mail: amit@nacad.ufrj.br

Bliman

Sorbonne Universités, Inria, UPMC Univ Paris 06, Lab. J.L. Lions UMR CNRS 7598, Paris, France and Escola de Matemática Aplicada, Fundação Getulio Vargas, Rio de Janeiro - RJ, Brazil. E-mail: pierre-alexandre.bliman@inria.fr

Niedu

Petrobras, S.A. E-mail: guiniedu@gmail.com

Pazos (corresponding author)

Department of Electronics and Telecommunication Engineering, State University of Rio de Janeiro, Rio de Janeiro - RJ, Brazil. Tel.: 55-21-2334-2165 and 55-21-2334-0565. E-mail: quini.coppe@gmail.com 


\section{Funding}

A. Bhaya's work was supported by a BPP grant, G. Niedu and F. Pazos were at UFRJ and supported by DS and PNPD fellowships, respectively, all of them from National Counsel of Technological and Scientific Development $(\mathrm{CNPq})$, while this paper was being written.

\section{Introduction}

The appearance of multi-core processors has motivated much recent interest in multi-thread computation, in which each thread is assigned some part of a larger computation and executes concurrently with other threads, each on its own core. All threads, however, have relatively fast access to a common memory, which is the source and destination of all data manipulated by the thread.

With the availability of ever larger on-chip memory and multicore processors that allow multi-thread programming, it is now possible to propose a new paradigm in which each thread, with access to a common memory, computes its own estimate of the solution to the whole problem (i.e., decomposition of the problem into subproblems is avoided) and the threads exchange information amongst themselves, this being the cooperative step. The design of a cooperative algorithm has the objective of ensuring that exchanged information is used by the threads in such a way as to reduce overall convergence time.

The idea of information exchange between two iterative processes was introduced into numerical linear algebra, in the context of linear systems, long before the advent of multicore processors by Brezinski [10] under the name of hybrid procedures, defined as (we quote) "a combination of two arbitrary approximate solutions with coefficients summing up to one...(so that) the combination only depends on one parameter whose value is chosen in order to minimize the Euclidean norm of the residual vector obtained by the hybrid procedure... The two approximate solutions which are combined in a hybrid procedure are usually obtained by two iterative methods". The objective of minimizing the residue is to accelerate convergence of the overall hybrid procedure (also see $[2,9]$ ). This idea was generalized and discussed in the context of distributed asynchronous computation in [6]. It is also worthy of note that the paradigm of cooperation between threads, thought of as an independent agents, in order to achieve some common objective, is also becoming popular in many areas such as control $[18,22,21]$.

Several iterative methods to solve the linear algebraic equation

$$
\mathbf{A x}=\mathbf{b}
$$

where $\mathbf{A} \in \mathbb{R}^{n \times n}$ is symmetric positive definite and $n$ is large, are well known. Solving (1) is equivalent to finding the minimizer of the strictly convex scalar function

$$
f(\mathbf{x})=\frac{1}{2} \mathbf{x}^{\top} \mathbf{A} \mathbf{x}-\mathbf{b}^{\top} \mathbf{x}
$$


since the unique minimizer of $f$ is $\mathbf{x}^{*}=\mathbf{A}^{-1} \mathbf{b}$.

Conjugate direction methods, based on minimization of (2), can be regarded as being intermediate between the method of steepest descent and Newton's method. They are motivated by the desire to accelerate the typically slow convergence associated with steepest descent while avoiding the information requirements associated with the evaluation and inversion of the Hessian [19, chap. 9].

The conjugate gradient algorithm (CG) is the most popular conjugate direction method. It was developed by Hestenes and Stiefel [17]. The algorithm minimizes the scalar function $f(\mathbf{x})$ along conjugate directions searched at each iteration; the convergence of the sequence of points $\mathbf{x}_{k}$ to the solution point $\mathbf{x}^{*}$ is produced after at most $n$ iterations in exact arithmetic. The residual vector is defined as

$$
\mathbf{r}_{k}=\mathbf{A} \mathbf{x}_{k}-\mathbf{b}
$$

and, given (2), clearly $\mathbf{r}_{k}=\nabla f\left(\mathbf{x}_{k}\right)$. In the CG algorithm, the residual vector $\mathbf{r}_{k}$ and a direction vector $\mathbf{d}_{k}$ are calculated at the $k$ th iteration,for every $k$.

O'Leary [23] developed a block CG method (B-CG) in which the conjugate directions and the residues are taken as columns of $n \times p$ matrices. The BCG algorithm was designed to handle multiple right-hand sides which form a matrix $\mathbf{B} \in \mathbb{R}^{n \times p}$, but it is also capable of accelerating the convergence of linear systems with a single right-hand side, for example for solving systems in which several eigenvalues are widely separated from the others. Several properties observed by the vectors in the CG algorithm continue to be valid for the matrices used in the B-CG algorithm, e.g. the conjugacy property between the matrix directions. Also, in exact arithmetic, the convergence of the B-CG algorithm to the solution matrix $\mathbf{X}^{*}$ occurs after at most $\left\lceil\frac{n}{p}\right\rceil$ iterations, which may involve less work than applying the CG algorithm $p$ times. Gutknecht [16] also analyzes block methods based on Krylov subspaces with multiple right hand sides.

Brezinski ([8, sec. 4] and [3]) developed a block CG algorithm called "multiparameter CG" (MPCG), which is essentially the B-CG with a single righthand side $\mathbf{b}$ and a single initial point $\mathbf{x}_{0}$. The authors of $[8,3]$ build on the pioneering work of [23], and provide some additional properties, particularly about its convergence. It should be noted that the B-CG algorithm as well as the MPCG algorithm were proposed in the context of a single processor, so issues of multiprocessor implementation, speed up and flop counts were not considered in $[23,8,3]$.

This paper revisits Brezinski's MPCG algorithm from a multi-thread perspective, calling it, in order to emphasize the new context, the Cooperative Conjugate Gradient (CCG) algorithm. The cooperation between threads resides in the fact that each thread now uses information that comes from the other threads, and, in addition, the descent and conjugate directions are updated simultaneously. The multi-thread implementation of the CCG algorithm aims to accelerate the time to convergence with respect to the B-CG and the MPCG algorithms. Preliminary versions of this paper are [5,4]. 
In $[13,14]$, Gu, Liu et al present a multithread CG method named "multiple search direction conjugate gradient method" (MSD-CG), and its preconditioned version (PMSD-CG). The method is midway between the CG method and the Block Jacobi method. It is based on a notion of subdomains or partitioning of the unknowns. In each iteration there is one search direction per subdomain that is zero in the vector elements that are associated with other subdomains [13, p. 1134]. The algorithm can be executed in parallel by a multicore processor. The problem is divided into smaller blocks, thus dividing the direction vectors and the residual vectors into smaller vectors to be calculated by each processor separately.

This paper is organized as follows. In section 2 the conjugate gradient algorithm, as well as some basic properties of the conjugate directions are presented. In section 3 the cooperative conjugate gradient algorithm in a multithread context is presented. Their basic properties and the convergence rate are studied. In section 4, the computational complexity of the CCG algorithm, as well as the classic CG, the MPCG, and the MSD-CG are investigated. In section 5 experimental results are presented. In section 6 some general conclusions are mentioned. Finally, an appendix presents the proofs of theorems and lemmas.

\section{Preliminaries on the classical CG algorithm}

This section recalls basic results on the classical CG algorithm in order to motivate the presentation of the corresponding results for the cooperative CG algorithm. The reader is referred to $[19,15]$ for all proofs and further details on the CG algorithm.

Definition 1 Given a symmetric positive definite matrix $\mathbf{A}$, two nonzero vectors $\mathbf{d}_{1}$ and $\mathbf{d}_{2}$ are said to be $\mathbf{A}$-orthogonal, or $\mathbf{A}$-conjugate, if $\mathbf{d}_{1}^{\top} \mathbf{A d}_{2}=0$.

Lemma 1 If a set of nonzero vectors $\left\{\mathbf{d}_{0}, \ldots, \mathbf{d}_{k}\right\}$ are $\mathbf{A}$-conjugate (with respect to a positive definite matrix $\mathbf{A})$, then these vectors are linearly independent. The solution $\mathbf{x}^{*} \in \mathbb{R}^{n}$ of the system $\mathbf{A} \mathbf{x}=\mathbf{b}$ can be expressed as a linear combination of $n \mathbf{A}$-conjugate vectors $\left\{\mathbf{d}_{0}, \ldots, \mathbf{d}_{n-1}\right\}$.

$$
\mathbf{x}^{*}=\alpha_{0} \mathbf{d}_{0}+\cdots+\alpha_{n-1} \mathbf{d}_{n-1}
$$

where $\alpha_{i}=\frac{\mathbf{d}_{i}^{\top} \mathbf{b}}{\mathbf{d}_{i}^{\top} \mathbf{A d} \mathbf{d}_{i}}$ for all $i \in\{0, \ldots, n-1\}$.

Theorem 1 Let $\left\{\mathbf{d}_{0}, \ldots, \mathbf{d}_{n-1}\right\}$ be a set of $n \mathbf{A}$-conjugate nonzero vectors. For any $\mathbf{x}_{0} \in \mathbb{R}^{n}$, the sequence generated according to

$$
\mathbf{x}_{k+1}=\mathbf{x}_{k}+\alpha_{k} \mathbf{d}_{k}
$$

with

$$
\alpha_{k}=-\frac{\mathbf{r}_{k}^{\top} \mathbf{d}_{k}}{\mathbf{d}_{k}^{\top} \mathbf{A} \mathbf{d}_{k}}
$$

where $\mathbf{r}_{k}=\nabla f\left(\mathbf{x}_{k}\right)=\mathbf{A} \mathbf{x}_{k}-\mathbf{b}$, converges to the unique solution of $\mathbf{A} \mathbf{x}=\mathbf{b}$, $\mathbf{x}^{*}$ after $n$ steps, that is $\mathbf{x}_{n}=\mathbf{x}^{*}$. 
It is notable that the choice (5) ensures the convergence of the sequence (4) in at most $n$ steps in exact arithmetic, which is known as the finite termination property. However, one of the most interesting and still partially understood property of the CG algorithm is that, even when implemented in finite precision arithmetic, approximate convergence to standard tolerances occurs much faster than $n$ iterations [20]. Nevertheless, we will use this "worst case" estimate of time to convergence in order to generate flop count estimates of the CCG algorithm in section 4. Another fundamental result, the CCG analog of which is presented as Theorem 4 below, is called the expanding subspace theorem [19].

Theorem 2 Let $\mathcal{B}_{k}$ the space spanned by the set of nonzero conjugate vectors $\left\{\mathbf{d}_{0}, \ldots, \mathbf{d}_{k-1}\right\}$. The point $\mathbf{x}_{k}$ calculated by the sequence (4) with the step sizes (5) is the global minimizer of $f(\mathbf{x})$ on the subspace $\mathbf{x}_{0}+\mathcal{B}_{k}$. Moreover, the residual vector $\mathbf{r}_{k}=\nabla f\left(\mathbf{x}_{k}\right)=\mathbf{A} \mathbf{x}_{k}-\mathbf{b}$ is orthogonal to $\mathcal{B}_{k}$.

\subsection{The Conjugate Gradient algorithm}

The Conjugate Gradient method, developed by Hestenes and Stiefel [17], is the particular method of conjugate directions obtained when constructing the conjugate directions by Gram-Schmidt orthogonalization, achieved at step $k+1$ on the set of the gradients $\left\{\mathbf{r}_{0}, \ldots, \mathbf{r}_{k}\right\}$. A key point here is that this construction can be carried out iteratively. The conjugate gradient algorithm is based on the minimization at each iteration of the scalar function $f(\mathbf{x})$ on conjugate directions which form a basis of the Krylov subspace $\mathcal{K}_{k}\left(\mathbf{A}, \mathbf{r}_{0}\right):=\operatorname{span}\left\{\mathbf{r}_{0}, \mathbf{A} \mathbf{r}_{0}, \mathbf{A}^{2} \mathbf{r}_{0}, \ldots, \mathbf{A}^{k-1} \mathbf{r}_{0}\right\}$.

The algorithm is described as follows. Starting from any $\mathbf{x}_{0} \in \mathbb{R}^{n}$, and choosing $\mathbf{d}_{0}=\mathbf{r}_{0}=\mathbf{A} \mathbf{x}_{0}-\mathbf{b}$, at each iteration, calculate:

$$
\begin{array}{r}
\mathbf{x}_{k+1}=\mathbf{x}_{k}+\alpha_{k} \mathbf{d}_{k} \\
\alpha_{k}=-\frac{\mathbf{r}_{k}^{\top} \mathbf{d}_{k}}{\mathbf{d}_{k}^{\top} \mathbf{A} \mathbf{d}_{k}} \\
\mathbf{d}_{k+1}=\mathbf{r}_{k+1}+\beta_{k} \mathbf{d}_{k} \\
\beta_{k}=-\frac{\mathbf{r}_{k+1}^{\top} \mathbf{A} \mathbf{d}_{k}}{\mathbf{d}_{k}^{\top} \mathbf{A} \mathbf{d}_{k}}
\end{array}
$$

In order to qualify as a conjugate gradient algorithm, the directions $\mathbf{d}_{k}$ generated at each step should be $\mathbf{A}$-conjugate, which is confirmed in the following theorem.

Theorem 3 (Conjugate Gradient Theorem) The conjugate gradient algorithm (6)-(9) is a conjugate direction method. If it does not terminate at the step $k$, then:

1. $\operatorname{span}\left\{\mathbf{r}_{0}, \mathbf{A r}_{0}, \ldots ; \mathbf{A}^{k} \mathbf{r}_{0}\right\}=\operatorname{span}\left\{\mathbf{r}_{0} ; \ldots ; \mathbf{r}_{k}\right\}=\operatorname{span}\left\{\mathbf{d}_{0}, \ldots, \mathbf{d}_{k}\right\}$. These subspaces have dimension $k+1$.

2. $\mathbf{d}_{k}^{\top} \mathbf{A} \mathbf{d}_{i}=0, \quad \forall i<k$. 
3. $\mathbf{r}_{k}^{\top} \mathbf{r}_{i}=0, \forall i<k$.

4. the point $\mathbf{x}_{k+1}$ is the minimizer of $f(\mathbf{x})$ on the affine subspace $\mathbf{x}_{0}+\operatorname{span}\left\{\mathbf{d}_{0} ; \ldots ; \mathbf{d}_{k}\right\}$

5. $\alpha_{k}=\frac{\mathbf{r}_{k}^{\top} \mathbf{r}_{k}}{\mathbf{d}_{k}^{\top} \mathbf{A d} d_{k}}$.

6. $\beta_{k}=\frac{\mathbf{r}_{k+1}^{T} \mathbf{r}_{k+1}}{\mathbf{r}_{k}^{\top} \mathbf{r}_{k}}$.

For a proof of this theorem as well as further details on the contents of this section, see [19, chap. 9] and [15, chap. 14].

When the residue vector is zero, the optimum has been attained, showing that $\mathrm{CG}$ terminates in finite time, in exact arithmetic. Some other results about the convergence of the algorithm (6)-(9) can be found in [7]. Interesting properties, both as an algorithm in exact arithmetic and as one in finite precision arithmetic can be found in $[20,12]$.

\section{The Cooperative Conjugate Gradient method}

Note that the conjugate gradient method is not parallelizable, because calculation of the new direction $\mathbf{d}_{k+1}$ requires the new residue $\mathbf{r}_{k+1}$ to have been calculated first.

However, if we suppose that $p$ initial conditions are used, then we may ask if it is possible to initiate $p$ CG-like computations in parallel and, in addition, share information amongst the $p$ processors carrying out these computations in such a way that there is an overall reduction in time of convergence? We shall refer to the $p$ processors as threads, in order that the cooperative computation paradigm that we introduce below have a natural interpretation as a multithread cooperative algorithm.

The extension of the method (6)-(9) using $p$ threads is defined using the following matrices:

1. $\mathbf{X}:=\left[\begin{array}{lll}\mathbf{x}_{1} & \mathbf{x}_{2} \cdots \mathbf{x}_{p}\end{array}\right] \in \mathbb{R}^{n \times p}$ is the matrix of solution estimates, in which the $i$ th column is assigned to the $i$ th thread.

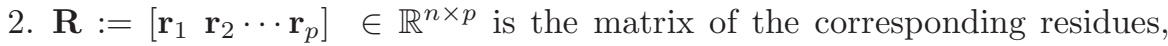
such that $\mathbf{r}_{i}=\mathbf{A} \mathbf{x}_{i}-\mathbf{b}, \forall i \in\{1, \ldots, p\}$.

3. $\mathbf{D}:=\left[\begin{array}{ll}\mathbf{d}_{1} & \mathbf{d}_{2} \cdots \mathbf{d}_{p}\end{array}\right] \in \mathbb{R}^{n \times p}$ is the matrix of the corresponding descent directions.

From an initial matrix $\mathbf{X}_{0}$, with residue $\mathbf{R}_{0}=\mathbf{A} \mathbf{X}_{0}-\mathbf{b} \mathbf{1}_{p}^{\top}$, and initial directions chosen as $\mathbf{D}_{0}=\mathbf{R}_{0}$, at each step calculate:

$$
\begin{array}{r}
\mathbf{X}_{k+1}=\mathbf{X}_{k}+\mathbf{D}_{k} \boldsymbol{\alpha}_{k}^{\top} \\
\mathbf{D}_{k+1}=\mathbf{R}_{k+1}+\mathbf{D}_{k} \boldsymbol{\beta}_{k}^{\top}
\end{array}
$$

where,

$$
\begin{aligned}
\boldsymbol{\alpha}_{k}=-\mathbf{R}_{k}^{\top} \mathbf{D}_{k}\left(\mathbf{D}_{k}^{\top} \mathbf{A} \mathbf{D}_{k}\right)^{-1} & \in \mathbb{R}^{p \times p} \\
\boldsymbol{\beta}_{k}=-\mathbf{R}_{k+1}^{\top} \mathbf{A D}_{k}\left(\mathbf{D}_{k}^{\top} \mathbf{A} \mathbf{D}_{k}\right)^{-1} & \in \mathbb{R}^{p \times p}
\end{aligned}
$$


until a stopping criterion (for example $\left\|\mathbf{r}_{i}\right\|$ smaller than a given tolerance for some $i \in\{1, \ldots, p\})$ is met. In the following, we shall assume that $\mathbf{D}_{k}$ is a full column rank matrix for all iterations $k$. Note that if $p=1$, the method (10)-(13) coincides with (6)-(9).

\section{Remark 1}

(a) O'Leary [23] considers a block-CG solver which treats multiple right hand sides at once $\left(\mathbf{B} \neq \mathbf{b} \mathbf{1}_{p}^{\top}\right)$. Brezinski $([8$, sec. 04] and [3]) considers only one thread $\mathbf{x}$ and the initial matrix $\mathbf{R}_{0}=\mathbf{D}_{0}$ is a particular partition of the initial residue such that $\mathbf{R}_{0} \mathbf{1}_{p}=\mathbf{r}_{0}=\mathbf{A} \mathbf{x}_{0}-\mathbf{b}$.

(b) In section 3.2, the case where $p$ does not necessarily divide $n$ and the case where rank degeneracy may occur are both analysed: neither case is considered in $[8,3]$.

(c) A preconditioned version of a multiparameter CG algorithm (MPCG) was presented in [11] which proposes a matrix version of the Gram-Schmidt process to find the conjugate direction matrices. However, this procedure is very expensive in computational terms. A preconditioned version of the CCG algorithm is not studied here, and will be the object of future research. It is expected that the advantages obtained by preconditioning the classical CG algorithm will also hold for the CCG algorithm.

\subsection{Properties of the cooperative conjugate gradient method}

All the relevant results on the CCG algorithm are collected in this subsection and the next subsection, while the proofs of the key properties are in an appendix, in order for this paper to be complete and self-contained. Lemmas 6, 7 and theorems 5, 6 are new, to the best of our knowledge.

In order to present CCG properties that are analogous to those presented in section 2.1, some notation is introduced. For any set of vectors $\mathbf{r}_{i} \in \mathbb{R}^{n}, i \in$ $\{0, \ldots, k\}$, we denote respectively $\left\{\mathbf{r}_{i}\right\}_{0}^{k}$ and $\left[\mathbf{r}_{i}\right]_{0}^{k}$ the set of these vectors and the matrix obtained by their concatenation: $\left[\mathbf{r}_{i}\right]_{0}^{k}=\left[\mathbf{r}_{0} \mathbf{r}_{1} \cdots \mathbf{r}_{k}\right] \in \mathbb{R}^{n \times(k+1)}$. The notation span $\left[\mathbf{r}_{i}\right]_{0}^{k}$ will denote the subspace of linear combination of the columns of the matrix $\left[\mathbf{r}_{i}\right]_{0}^{k}$. When $\mathbb{R}^{n}$ is the ambient vector space, we have

$$
\operatorname{span}\left[\mathbf{r}_{i}\right]_{0}^{k}=\left\{\mathbf{v} \in \mathbb{R}^{n} \mid \exists \boldsymbol{\gamma} \in \mathbb{R}^{k+1}, \mathbf{v}=\sum_{i=0}^{k} \gamma_{i} \mathbf{r}_{i}=\left[\mathbf{r}_{i}\right]_{0}^{k} \boldsymbol{\gamma}\right\}
$$

Similarly, for matrices $\mathbf{R}_{i} \in \mathbb{R}^{n \times p}, i \in\{0, \ldots, k\}$, we introduce the notations $\left\{\mathbf{R}_{i}\right\}_{0}^{k}$ and $\left[\mathbf{R}_{i}\right]_{0}^{k}$, which denote, respectively, the set of these matrices and the matrix obtained as concatenation of the matrices $\mathbf{R}_{0}, \mathbf{R}_{1}, \ldots, \mathbf{R}_{k}$, that is $\left[\mathbf{R}_{i}\right]_{0}^{k}=\left[\mathbf{R}_{0} \mathbf{R}_{1} \cdots \mathbf{R}_{k}\right] \in \mathbb{R}^{n \times p(k+1)}$. Also, we write span $\left[\mathbf{R}_{i}\right]_{0}^{k}$ for the subspace obtained by all possible linear combinations of the columns of $\left[\mathbf{R}_{i}\right]_{0}^{k}$ :

$$
\operatorname{span}\left[\mathbf{R}_{i}\right]_{0}^{k}=\left\{\mathbf{v} \in \mathbb{R}^{n} \mid \exists \boldsymbol{\gamma} \in \mathbb{R}^{(k+1) p}, \mathbf{v}=\left[\mathbf{R}_{i}\right]_{0}^{k} \boldsymbol{\gamma}\right\}
$$


Definition 2 Two matrices $\mathbf{Q}_{1}$ and $\mathbf{Q}_{2}$ are called orthogonal if $\mathbf{Q}_{1}^{\top} \mathbf{Q}_{2}=0$, and they are called $\mathbf{A}$-conjugate, or simply conjugate with respect to a matrix $\mathbf{A}$ if $\mathbf{Q}_{1}^{\top} \mathbf{A} \mathbf{Q}_{2}=0$.

To prove the properties of the algorithm (10)-(13) we first substitute the equation (11) with step size (13) by the direction matrices generated by the GramSchmidt process, defined as follows.

The Gram-Schmidt process generates conjugate directions sequentially [15, p. 389]. It can be extended to work with matrices, where every column of a matrix generated by the process in a step is conjugate with respect to every column of the matrices generated in the former steps, in the following way:

$$
\mathbf{D}_{k+1}=\mathbf{R}_{k+1}-\sum_{j=0}^{k} \mathbf{D}_{j}\left(\mathbf{D}_{j}^{\top} \mathbf{A D}_{j}\right)^{-1} \mathbf{D}_{j}^{\top} \mathbf{A R}_{k+1}, \quad \mathbf{D}_{0}=\mathbf{R}_{0}
$$

To iterate using (14), it is assumed that all the matrices generated at each iteration have full column rank (so $\mathbf{D}_{j}^{\top} \mathbf{A} \mathbf{D}_{j}$ is nonsingular). It is easy to see that (14) generates matrices such that $\mathbf{D}_{j}^{\top} \mathbf{A D}_{i}=0$ for all $i, j \in\{0, \ldots, k+1\}$, $i \neq j$. As in the classical case, iteration (14) is expensive in computational terms.

Theorem 4 Let the direction matrices $\mathbf{D}_{0}, \ldots, \mathbf{D}_{k}$ be conjugate and of full column rank. The columns of $\mathbf{X}_{k}$ calculated as in (10), using the step size (12), minimize $f\left(\mathbf{x}_{i_{k}}\right), \forall i \in\{1, \ldots, p\}$ on the affine set $\mathbf{x}_{i_{0}}+\operatorname{span}\left[\mathbf{D}_{j}\right]_{0}^{k-1}$. Moreover, the columns of $\mathbf{R}_{k}$ are orthogonal to span $\left[\mathbf{D}_{j}\right]_{0}^{k-1}$, which means that $\mathbf{R}_{k}^{\top} \mathbf{D}_{j}=0, \forall j<k$.

The next lemma is easy to prove, by induction using (10)-(13), and the fact that $\mathbf{R}_{k+1}=\mathbf{A} \mathbf{X}_{k+1}-\mathbf{b} \mathbf{1}_{p}^{\top}=\mathbf{R}_{k}+\mathbf{A} \mathbf{D}_{k} \boldsymbol{\alpha}_{k}^{\top}$.

Lemma 2 Suppose that $\mathbf{D}_{0}=\mathbf{R}_{0}$, then:

$$
\operatorname{span}\left[\mathbf{R}_{0} \mathbf{R}_{1} \cdots \mathbf{R}_{k}\right]=\operatorname{span}\left[\mathbf{R}_{0} \mathbf{A} \mathbf{R}_{0} \cdots \mathbf{A}^{k} \mathbf{R}_{0}\right]=\operatorname{span}\left[\mathbf{D}_{0} \mathbf{D}_{1} \cdots \mathbf{D}_{k}\right]
$$

Gutknecht [16, sec. 8] defines block-Krylov subspace generated by matrices $\mathbf{A} \in \mathbb{R}^{n \times n}$ and $\mathbf{R}_{0} \in \mathbb{R}^{n \times p}$ as

$$
\mathcal{K}_{k}^{\square}\left(\mathbf{A}, \mathbf{R}_{0}\right):=\left\{\mathbf{X} \in \mathbb{R}^{n \times p} \mid \exists \gamma_{0}, \cdots, \gamma_{k} \in \mathbb{R}^{p \times p}, \mathbf{X}=\sum_{i=0}^{k-1} \mathbf{A}^{i} \mathbf{R}_{0} \gamma_{i}\right\}
$$

and a block-Krylov subspace method as an iterative method which generates matrices belonging to a block-Krylov subspace $\mathbf{X}_{k} \in \mathbf{X}_{0}+\mathcal{K}_{k}^{\square}\left(\mathbf{A}, \mathbf{R}_{0}\right)$, at each iteration.

According to these definitions the method (10) with step size (12) is a block-Krylov subspace method. Note, however, that the spaces defined in (15) are not block-Krylov subspaces.

Lemma 3 The matrices generated by (14) are the same as those generated by (11) with step size (13). 
The following useful lemmas are proved in $[23,8,3]$.

\section{Lemma 4 (Orthogonality properties)}

$$
\begin{array}{r}
\mathbf{R}_{k}^{\top} \mathbf{A D}_{k}=\mathbf{D}_{k}^{\top} \mathbf{A D}_{k} \\
\mathbf{R}_{k}^{\top} \mathbf{R}_{k}=\mathbf{R}_{k}^{\top} \mathbf{D}_{k}
\end{array}
$$

Lemma 5 (Formulas for matrix step sizes)

$$
\begin{array}{r}
\boldsymbol{\alpha}_{k}^{\top}=-\left(\mathbf{D}_{k}^{\top} \mathbf{A D}_{k}\right)^{-1} \mathbf{R}_{k}^{\top} \mathbf{R}_{k} \\
\boldsymbol{\beta}_{k}^{\top}=-\left(\mathbf{R}_{k}^{\top} \mathbf{R}_{k}\right)^{-1} \mathbf{R}_{k+1}^{\top} \mathbf{R}_{k+1}
\end{array}
$$

Theorem 4, and lemmas 2 and 3 indicate that, as long as the residue matrix $\mathbf{R}_{k}$ is full rank, the algorithm CCG behaves essentially as does CG, providing $p$ different estimates at iteration $k$, each of them being optimal in an affine set constructed from one of the $p$ initial conditions and the common vector space obtained from the columns of the direction matrices $\mathbf{D}_{i}, i \in\{0, \ldots, k-1\}$. This vector space, span $\left[\mathbf{D}_{i}\right]_{0}^{k}$, has dimension $(k+1) p$ : each iteration involves the cancellation of $p$ directions. Notice that different columns of the matrices $\mathbf{D}_{k}$ are not necessarily $\mathbf{A}$-orthogonal (in other words, $\mathbf{D}_{k}^{\top} \mathbf{A} \mathbf{D}_{k}$ is not necessarily diagonal), but, when $\mathbf{R}_{k}$ is full rank, they constitute a set of $p$ independent vectors. The statements as well as the proofs of these theorems and lemmas (all in the appendix) have been inspired by the corresponding ones for the conventional CG algorithm given in [19, p. 270] and [15, pp. 390-391].

\subsection{Convergence of the cooperative conjugate gradient method}

To prove the convergence of the algorithm (10)-(13), first consider the case in which, if rank $\mathbf{D}_{k}=p$, then rank $\mathbf{D}_{k+1}=p$, at least until $\mathbf{D}_{k+1}=\mathbf{R}_{k+1}=\mathbf{0}$, in which case the algorithm, in exact arithmetic, terminates.

Lemma 6 If rank $\mathbf{D}_{0}=p$ and the algorithm (10)-(13) does not terminate at the iteration $k$, which implies that $\mathbf{R}_{k}$ and $\mathbf{D}_{k}$ are different from zero, then rank $\left[\mathbf{D}_{i}\right]_{0}^{k-1}=p k \leq n$, which means that, before convergence, all the column vectors are linearly independent.

Remark 2 If rank $\left[\mathbf{D}_{i}\right]_{0}^{k}=n<p(k+1)$, then $\mathbf{D}_{k}^{\top} \mathbf{A D}_{\ell} \neq 0, \forall \ell<k$, which implies that the conjugacy property is no longer satisfied. In fact, only the $n-p k$ first columns of the matrix $\mathbf{D}_{k}$ continue to be conjugate to the former matrices, that is $\mathbf{D}_{\ell}^{\top} \mathbf{A}\left[\mathbf{d}_{i_{k}}\right]_{1}^{n-p k}=0, \forall \ell<k$.

The same happens with the matrix $\mathbf{R}_{k}$, where $\mathbf{R}_{k}^{\top} \mathbf{D}_{\ell} \neq 0, \forall \ell<k$, only the first $n-p k$ columns continue to be orthogonal to span $\left[\mathbf{D}_{i}\right]_{0}^{k-1}$, that is $\mathbf{D}_{\ell}^{\top}\left[\mathbf{r}_{i_{k}}\right]_{1}^{n-p k}=0, \forall \ell<k$.

The following theorem affirms that, in the iteration that follows the satisfaction of this condition (i.e., iteration $k+1$ such that $p(k+1)>n$ ), convergence to the solution occurs. We first consider the simpler case where $p$ divides $n$. 
Theorem 5 If rank $\mathbf{R}_{0}=p$, then all of the threads in the cooperative conjugate gradient method (10)-(13) converge to the solution $\mathbf{x}^{*}$ in at most $k^{*}=\frac{n}{p}$ iterations, which means that $\mathbf{R}_{k^{*}}=\mathbf{D}_{k^{*}}=0$ and $\mathbf{X}_{k^{*}}=\mathbf{x}^{*} \mathbf{1}_{p}^{\top}$.

Note that lemma 6 indicates that if each matrix $\mathbf{D}_{i}$ generated at each iteration has a rank $p$, then all the columns of $\left[\mathbf{D}_{0} \cdots \mathbf{D}_{k}\right]$ are linearly independent. Unfortunately, even if all columns of the matrices $\mathbf{R}_{k+1}$ and $\mathbf{D}_{k}$ are linearly independent, this does not guarantee that the columns of $\mathbf{D}_{k+1}$, calculated by (11), also has linearly independent columns.

When the columns of $\mathbf{D}_{k}$ are linearly dependent, it is enough to eliminate columns (threads) in such a way that $\mathbf{D}_{k}$ continues to have full column rank, choosing any full-rank subset of columns (so that $\mathbf{D}_{k}^{\top} \mathbf{A} \mathbf{D}_{k}$ continues to be nonsingular). The linear dependence of the columns of $\mathbf{D}_{k}$ is known as rank degeneracy (or deflation, which is the term used in [16, sec. 8]). Note that the term rank degeneracy includes the case when $p$ does not divide $n$ and thus $p k^{*}>n$, as pointed out in remark 2 .

O'Leary also considers the possibility of "deleting the zero or redundant column $j$ of $\mathbf{D}_{k}$ and the corresponding columns of $\mathbf{X}_{k}$ and $\mathbf{R}_{k}$, and continuing the algorithm with $p-1$ vectors... The resulting sequences retain all of the properties necessary to guarantee convergence" [23, p. 301].

In order to consider the case where rank degeneracy occurs, denote as $p_{k}$ the number of threads such that rank $\mathbf{D}_{k}=p_{k}$. We assume $p_{0}=p$, and thus $p_{k} \leq p$ for all $k>0$.

Note that the best case is $p_{k}=p$ for all $k>0$ until convergence occurs (there is no rank degeneracy). The worst case is $p_{k}=1, \forall k>0$ until convergence occurs.

Lemma 7 There exists a finite natural number $k^{*}$ such that

$$
\min _{k^{*} \in \mathbb{N}} \operatorname{rank}\left[\mathbf{D}_{i}\right]_{0}^{k^{*}-1}=n
$$

The following theorem states conditions for convergence of the CCG algorithm in the general case where rank degeneracy may occur.

Theorem 6 If rank $\mathbf{R}_{0}=p>1$, all the threads that converge in the conjugate gradient method (10)-(13) converge to $\mathbf{x}^{*}$ in $\left\lceil\frac{n}{p}\right\rceil \leq k^{*} \leq n-p+1$ iterations, i.e. $\mathbf{x}_{i_{k^{*}}}=\mathbf{x}^{*}$ and $\mathbf{r}_{i_{k^{*}}}=\mathbf{d}_{i_{k^{*}}}=\mathbf{0}$ for all $i \in\left\{1, \ldots, p_{k^{*}-1}\right\}$.

The proof of this theorem is similar to that of theorem 5 for all the threads that are not eliminated at any iteration by rank degeneracy, i.e. $\mathbf{x}_{i_{k}}, \forall i \in$ $\left\{1, \ldots, p_{k^{*}-1}\right\}$. Note that, although rank degeneracy may occur, for all $i, j \in$ $\left\{0, \ldots, k^{*}-1\right\}, i \neq j, \mathbf{D}_{i} \in \mathbb{R}^{n \times p_{i}}, \mathbf{D}_{j} \in \mathbb{R}^{n \times p_{j}}$, the conjugacy property $\mathbf{D}_{i}^{\top} \mathbf{A D}_{j}=0 \in \mathbb{R}^{p_{i} \times p_{j}}$ continues to be valid, as well as the orthogonality property $\mathbf{R}_{i}^{\top} \mathbf{D}_{j}=0 \in \mathbb{R}^{p_{i} \times p_{j}}, \forall j<i$.

The following lemma, proved in [3, property 11] and [23, theorem 5], gives the rate of convergence of the CCG algorithm. 
Table 1 Example of the execution of the CCG algorithm with a randomly generated matrix $\mathbf{A} \in \mathbb{R}^{50 \times 50}$ from initial conditions that are the columns of a randomly generated matrix $\mathbf{X}_{0} \in \mathbb{R}^{50 \times 6}$, which implies the use of $p=6$ threads. The right hand side $\mathbf{b}$ is also random. The norm of the residual vector of each thread and the rank of the matrices $\left[\mathbf{D}_{k}\right]$ and $\left[\mathbf{D}_{i}\right]_{0}^{k}$ at each iteration $k$ are reported.

\begin{tabular}{|c||c|c|c|c|c|c|c|c|}
\hline $\mathrm{k}$ & $\operatorname{rank}\left[\mathbf{D}_{k}\right]$ & $\operatorname{rank}\left[\mathbf{D}_{i}\right]_{0}^{k}$ & $\left\|\mathbf{r}_{1_{k}}\right\|$ & $\left\|\mathbf{r}_{2_{k}}\right\|$ & $\left\|\mathbf{r}_{3_{k}}\right\|$ & $\left\|\mathbf{r}_{4_{k}}\right\|$ & $\left\|\mathbf{r}_{5_{k}}\right\|$ & $\left\|\mathbf{r}_{6_{k}}\right\|$ \\
\hline \hline 0 & 6 & 6 & $3.8010^{5}$ & $4.1610^{5}$ & $3.8310^{5}$ & $3.4410^{5}$ & $3.8210^{5}$ & $3.0810^{5}$ \\
\hline 1 & 6 & 12 & $1.0410^{5}$ & $0.8810^{5}$ & $1.1110^{5}$ & $1.0910^{5}$ & $0.9710^{5}$ & $0.9310^{5}$ \\
\hline 2 & 6 & 18 & $4.4310^{4}$ & $4.1910^{4}$ & $4.1810^{4}$ & $3.0410^{4}$ & $3.9810^{4}$ & $4.4410^{4}$ \\
\hline 3 & 6 & 24 & $1.2810^{4}$ & $2.0210^{4}$ & $2.1510^{4}$ & $1.7810^{4}$ & $1.8510^{4}$ & $2.3910^{4}$ \\
\hline 4 & 6 & 30 & $5.9410^{3}$ & $8.6910^{3}$ & $7.4810^{3}$ & $12.5210^{3}$ & $14.4810^{3}$ & $10.8010^{3}$ \\
\hline 5 & 6 & 36 & $3.1810^{3}$ & $3.1110^{3}$ & $4.0410^{3}$ & $5.5710^{3}$ & $6.0010^{3}$ & $4.6010^{3}$ \\
\hline 6 & 6 & 42 & $1.5010^{3}$ & $2.9610^{3}$ & $3.3110^{3}$ & $3.6010^{3}$ & $2.7010^{3}$ & $4.4210^{3}$ \\
\hline 7 & 6 & 48 & $1.1610^{3}$ & $4.0810^{3}$ & $3.5610^{3}$ & $4.4110^{3}$ & $2.4210^{3}$ & $5.5810^{3}$ \\
\hline 8 & 6 & 50 & 252.87 & 227.31 & 293.49 & 250.26 & 641.00 & 363.04 \\
\hline 9 & 6 & 50 & $1.0910^{-4}$ & $1.1310^{-4}$ & $1.4310^{-4}$ & $1.3610^{-4}$ & $2.6710^{-4}$ & $0.4510^{-4}$ \\
\hline
\end{tabular}

Lemma 8 Let $\kappa=\lambda_{n} / \lambda_{1}$ be the condition number of $A$ :

$$
\left\|\mathbf{x}_{i_{p_{k}}}-\mathbf{x}^{*}\right\|_{\mathbf{A}}=\frac{2}{\sqrt{\lambda_{1}}} \sum_{i=1}^{p}\left\|\mathbf{r}_{i_{0}}\right\|\left(\frac{\sqrt{\kappa}-1}{\sqrt{\kappa}+1}\right)^{k}
$$

Next, we present an example of the execution of the CCG algorithm for a dense randomly generated matrix and using six threads. In this example, no rank degeneracy occurs, so that the columns of the matrix $\left[\mathbf{D}_{i}\right]_{0}^{k}$ are linearly independent until the iteration $k^{*}$.

Example 1 Let $\mathbf{A} \in \mathbb{R}^{50 \times 50}$ be a randomly generated symmetric positive definite matrix, $\mathbf{b} \neq \mathbf{0} \in \mathbb{R}^{50}$, and 6 initial conditions $\mathbf{X}_{0} \in \mathbb{R}^{50 \times 6}$ are also randomly chosen; thus we have $p=6$ threads. Table 1 reports the rank of the matrices $\left[\mathbf{D}_{k}\right]$ and $\left[\mathbf{D}_{i}\right]_{0}^{k}$ and the norm of the residual vector of every thread at each iteration $k$. Note that in this example the direction vectors are linearly independent $\left(\left[\mathbf{D}_{i}\right]_{0}^{k}\right.$ has full column rank and hence no rank degeneracy occurs). The convergence is produced at an iteration $k^{*}=9$, where the norm of the residual vectors are lower than $10^{-3}$.

Brezinski [3, p. 12] affirms that, when $p k^{*}>n$, rank degeneracy always occurs in the last iteration, then a "breakdown occurs ... and no general conclusion can be deduced". In fact, Algorithm 1 shows pseudocode for the cooperative conjugate gradient algorithm in the case when rank degeneracy may occur. In algorithm 1, $\left.\mathbf{X}\right|_{j \in J}$ refers to the matrix $\mathbf{X}$ from which the columns specified in the set $J$ have been removed.

\section{Estimates of operation counts and speedup of the CCG algorithm}

This section estimates the operation counts and speedup of the CCG algorithm, when using the CCG algorithm with $p$ threads, instead of CG and MPCG algorithms. The expected speedup is due to the parallelism inherent in a multi-thread implementation. In order to calculate these estimates for 


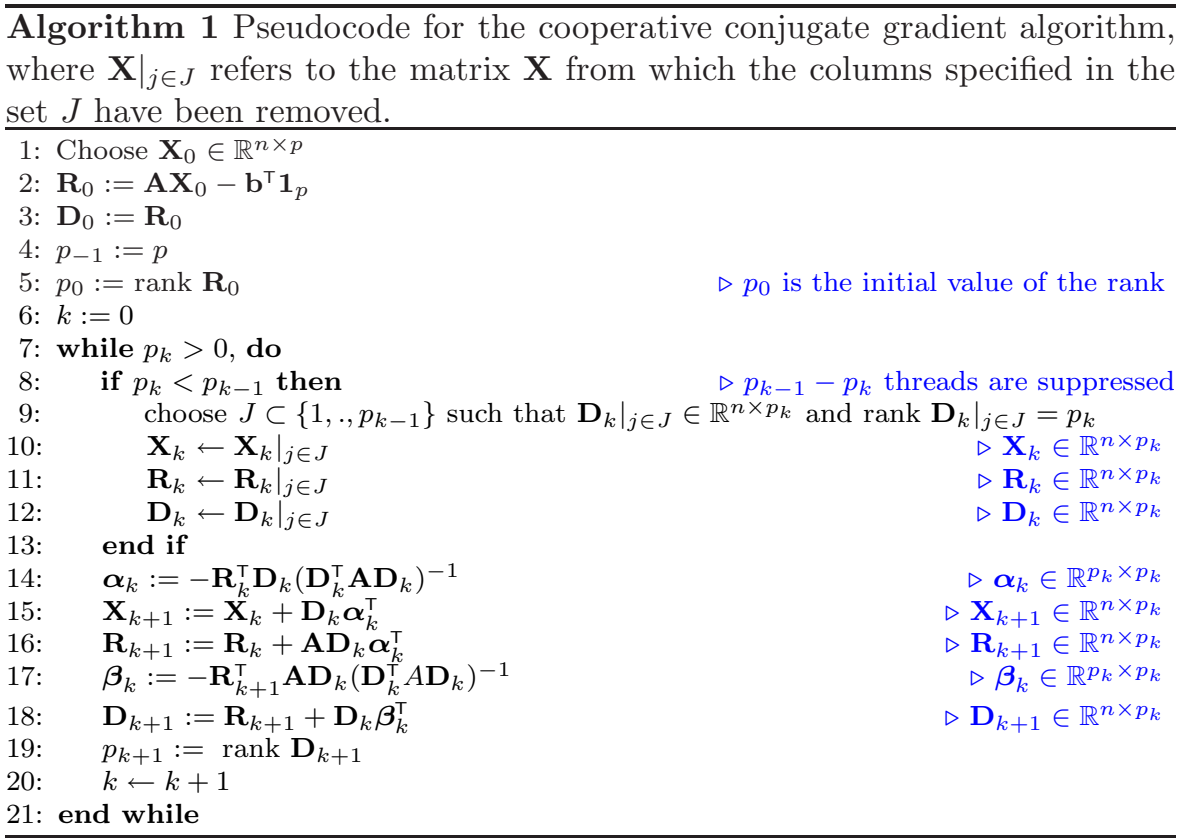

the cooperative conjugate gradient algorithm (10)-(13), we make the following assumptions:

1. the computations are carried out in exact arithmetic;

2. the only floating point operations that are counted are multiplication and division and both operations take the same amount of time;

3. the worst case of finite termination in $\left\lceil\frac{n}{p}\right\rceil$ steps occurs, where $n$ is the dimension of the matrix $\mathbf{A}$;

4. all threads are computationally identical: i.e., all floating point operations are executed in the same amount of time on each thread,

5. rank degeneracy does not occur, i.e. $p_{k}=p$ for all $k \in\left\{0, \ldots, k^{*}-1\right\}$.

Rewriting equations (10)-(13) to make the calculations in (10), (11) explicit, we assume that each iteration of the algorithm requires calculating the following recursions:

$$
\begin{aligned}
& \mathbf{X}_{k+1}=\mathbf{X}_{k}-\mathbf{D}_{k}\left(\mathbf{R}_{k}^{\top} \mathbf{D}_{k}\left(\mathbf{D}_{k}^{\top} \mathbf{A} \mathbf{D}_{k}\right)^{-1}\right)^{\top} \\
& \mathbf{R}_{k+1}=\mathbf{R}_{k}-\mathbf{A D}_{k}\left(\mathbf{R}_{k}^{\top} \mathbf{D}_{k}\left(\mathbf{D}_{k}^{\top} \mathbf{A} \mathbf{D}_{k}\right)^{-1}\right)^{\top} \\
& \mathbf{D}_{k+1}=\mathbf{R}_{k+1}-\mathbf{D}_{k}\left(\mathbf{R}_{k+1}^{\top} \mathbf{A} \mathbf{D}_{k}\left(\mathbf{D}_{k}^{\top} \mathbf{A} \mathbf{D}_{k}\right)^{-1}\right)^{\top}
\end{aligned}
$$

Table 2, based on the iteration defined in (21), shows the number of floating point operations realized per iteration by each processor in a multi-thread implementation. Total computation time can be assumed to be proportional to the total number of floating point operations required to satisfy the stopping criterion, neglecting the time spent on communication.

In Table 2, the first column indicates the task carried out at each stage by every thread. The double lines, separating the first row from the second 
Table 2 Operations and corresponding number of floating point operations in the iteration $k$ executed by each processor in a multi-thread implementation

\begin{tabular}{|c|c|c|c|c|c|}
\hline $\begin{array}{c}\text { operation } \\
\text { of proc. } i \text { (a) }\end{array}$ & result (b) & $\begin{array}{c}\text { dimension } \\
\text { of the result }\end{array}$ & products & additions & divisions \\
\hline $\mathrm{Ad}_{i}$ & $\mathrm{AD}$ & $n \times p$ & $n^{2}$ & $n(n-1)$ & 0 \\
\hline$\overline{\mathrm{d}} \mathrm{d}_{i}^{\mathrm{ADD}}$ & $\mathrm{D}^{1} \mathrm{AD}$ & $p \times p$ & $\overline{n p}$ & $p(n-1)$ & 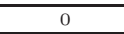 \\
\hline $\mathbf{r}_{i}^{1} \mathbf{D}$ & $\mathbf{R}^{\prime} \mathbf{D}$ & $p \times p$ & $n p$ & $p(n-1)$ & 0 \\
\hline $\boldsymbol{\alpha}_{i} \mid \boldsymbol{\alpha}_{i}\left(\mathbf{D}^{\top} \mathrm{AD}\right)=\mathbf{r}_{i}^{\top} \mathbf{D}$ & $\alpha:=\mathbf{R}^{\top} \mathbf{D}\left(\mathbf{D}^{\top} \mathbf{A D}\right)^{-1}$ & $p \times p$ & \multicolumn{2}{|c|}{$\frac{p(p+1)(2 p+1)}{6}-p$} & $\frac{p(p+1)}{2}$ (c) \\
\hline $\mathbf{r}_{i}:=\mathbf{r}_{i}-\mathbf{A D \alpha _ { i }}{ }_{i}^{\prime}$ & $\mathbf{R}:=\mathbf{R}-\mathbf{A D} \alpha$ & $n \times p$ & $n p$ & $n p$ & 0 \\
\hline $\mathbf{x}_{i}:=\mathbf{x}_{i}-\mathbf{D} \boldsymbol{\alpha}_{i}^{1}$ & $\mathrm{X}:=\mathrm{X}-\mathrm{D} \boldsymbol{\alpha}{ }^{1}$ & $n \times p$ & $n p$ & $n p$ & 0 \\
\hline $\mathbf{r}_{i}^{\prime} \mathrm{AD}$ & $\mathrm{R}^{1} \mathrm{AD}$ & $p \times p$ & $n p$ & $p(n-1)$ & 0 \\
\hline $\boldsymbol{\beta}_{i} \mid \boldsymbol{\beta}_{i}\left(\mathbf{D}^{\top} \mathbf{A D}\right)=\mathbf{r}_{i}^{\top}$ AD & $\boldsymbol{\beta}:=\mathbf{R}^{\top} \mathbf{A D}\left(\mathbf{D}^{\top} \mathbf{A D}\right)^{-1}$ & $p \times p$ & \multicolumn{2}{|c|}{$\frac{p(p+1)(2 p+1)}{6}-p$} & $\frac{p(p+1)}{2}$ (c) \\
\hline $\mathbf{d}_{i}:=\mathbf{r}_{i}-\mathbf{D} \boldsymbol{\beta}_{i}^{\mid}$ & $\mathbf{D}:=\mathbf{R}-\mathbf{D} \boldsymbol{\beta} \mid$ & $n \times p$ & $n p$ & $n p$ & 0 \\
\hline \multicolumn{3}{|c|}{$\begin{array}{l}\text { total number of products per iteration } \\
\text { total number of additions per iteration } \\
\text { total number of divisions per iteration }\end{array}$} & \multicolumn{3}{|c|}{$\begin{array}{c}n^{2}+6 n p+\frac{p(p+1)(2 p+1)}{3}-2 p \\
n^{2}-n+6 n p+\frac{p(p+1)(2 p+1)}{3}-5 p \\
p(p+1)\end{array}$} \\
\hline
\end{tabular}

(a) Operation realized by the $i$ th thread, for all $i \in\{1, \ldots, p\}$

(b) Composite result of the operations realized by all the $p$ threads at the same time

(c) These numbers of floating points operations are needed to realize a Gaussian elimination by LU factorization [25, p. 15] The double line indicates a stage at which communication between all threads occurs, which means that every thread $i$ need thow results from other threads.

and the second from the third, indicate the necessity of a phase of information exchange: every thread at that stage needs to know results from other threads. The second column, labelled composite result, contains the information that is available by pooling the partial results from each thread and the third column gives the dimension of this composite result. The last three columns contain the number of operations carried out by the $i^{\text {th }}$ thread.

As indicated by the last line of Table 2 , a total of $n^{2}+6 n p+\frac{p(p+1)(2 p+1)}{3}-2 p$ multiplications per processor is needed to complete an iteration. In addition, $p(p+1)$ divisions are carried out per iteration. Since, generically speaking, the algorithm ends in at most $\frac{n}{p}$ iterations, an estimate of the worst-case multithread execution time is given by the following result.

Theorem 7 (Worst-case multithread CCG flop count) The worst-case multithread execution of $C C G$ using $p$ agents for a linear system (1) of size $n$ requires

$$
N_{C C G}(p)=\frac{n^{3}}{p}+6 n^{2}+\frac{2}{3} n p^{2}+2 n p-\frac{2}{3} n
$$

multiplications and divisions carried out in parallel and synchronously, by each processor.

Note that (22) for $p=1$ gives the worst-case flop count for the CG algorithm:

$$
N_{C G}=N_{C C G}(1)=n^{3}+6 n^{2}+2 n
$$

multiplications and divisions.

Theorem 7 has the following important corollaries.

Corollary 1 (Multi-thread gain) For problems of size $n$ at least equal to 8 , it is always beneficial to use $p \leq n$ processors rather than a single one. In 
other words, when $n \geq 8$,

$$
\forall 1 \leq p \leq n, \quad N_{C C G}(1) \geq N_{C C G}(p)
$$

Proof:

$$
\begin{aligned}
N_{C C G}(1)-N_{C C G}(n) & =n^{3}+6 n^{2}+2 n-\left(\frac{2}{3} n^{3}+9 n^{2}-\frac{2}{3} n\right) \\
& =\frac{1}{3}\left(n^{3}-9 n^{2}+8 n\right) \\
& =\frac{1}{3} n(n-1)(n-8)
\end{aligned}
$$

Moreover,

$$
\left.\frac{d N_{C C G}}{d p}\right|_{p=1}=\frac{1}{3} n\left(-3 n^{2}+10\right)
$$

which is negative for $n \geq 2$, while

$$
\left.\frac{d N_{C C G}}{d p}\right|_{p=n}=n\left(\frac{4}{3} n+1\right)>0
$$

The convexity of $N_{C C G}(p)$ as a function of $p$ then yields the conclusion that $N_{C C G}(p) \leq N_{C C G}(1)$ for any $1 \leq p \leq n$.

Corollary 2 (Optimal multi-thread gain for CCG) For any size $n$ of the problem, there exists a unique optimal number $p^{*}$ of processors minimizing $N_{C C G}(p)$. Moreover, when $n \rightarrow+\infty$,

$$
\begin{gathered}
p^{*} \approx\left(\frac{3}{4}\right)^{\frac{1}{3}} n^{\frac{2}{3}} \\
N_{C C G}\left(p^{*}\right) \approx\left(\left(\frac{4}{3}\right)^{\frac{1}{3}}+\frac{2}{3}\left(\frac{3}{4}\right)^{\frac{2}{3}}\right) n^{2+\frac{1}{3}} \approx 1.651 n^{2+\frac{1}{3}}
\end{gathered}
$$

Proof:

$$
\frac{d N_{C C G}(p)}{d p}=-\frac{n^{3}}{p^{2}}+\frac{4}{3} n p+2 n
$$

There exists a unique $p^{*}$ such that $d N_{C C G} / d p=0$. For this value, one has $n^{2}=\left(p^{*}\right)^{2}\left(\frac{4}{3} p^{*}+2\right)$, which yields the asymptotic behavior given in (25a). The value in (25b) is directly deduced, by substituting (25a) in (22).

Corollary 1 implies that for $n>8$, for every choice of the number of threads $p: N_{C C G}(p)<N_{C G}$, and thus the CCG algorithm can be expected to converge in less time than the CG algorithm.

The important conclusion of corollary 2 is that, in the asymptotic limit, as $n$ becomes large, implying that the optimal $p^{*}$ also increases according to (25a), solution of $\mathbf{A x}=\mathbf{b}$ is possible, by the multi-thread method proposed in this paper, with a cost of $O\left(n^{2+\frac{1}{3}}\right)$ floating point operations, showing a clear advantage over the classical result of $O\left(n^{3}\right)$ for Gaussian elimination. 
4.1 Worst-case operation count for other block CG algorithms

The multiparameter conjugate gradient algorithm proposed by Brezinski in [3] and $[8$, sec. 4$]$, carried out using only one thread, calculates a total number of scalar products and scalar divisions given by

$$
N_{M P C G}(p)=p N_{C C G}(p)=n^{3}+6 n^{2} p+\frac{2}{3} n p^{3}+2 n p^{2}-\frac{2}{3} n p
$$

so that with $p>1, N_{C C G}<N_{M P C G}$, which means that the CCG algorithm implemented in a multi-thread context is faster than the MPCG algorithm. The fact that a lower number of iterations than the worst case $n / p$ is expected in practice does not modify the analysis, because for the same number of iterations to reach convergence for both the algorithms, the total number of floating point operations executed by the CCG algorithm is always smaller than those executed by the MPCG algorithm.

We emphasize that, in practice, specially when sparse matrices are used, the number of iterations to attain a specified error tolerance is usually much lower than $\left\lceil\frac{n}{p}\right\rceil$ and, furthermore, this behavior is also observed with the MPCG and the B-CG algorithms [20], just as is the case with the classical CG algorithm.

The multiple search direction conjugate gradient algorithm (MSD-CG) [13, 14], also implemented in a multi-thread context, performs a number of scalar products per thread and per iteration equal to $\frac{n^{2}}{p}+3 \frac{n}{p}+3 n+\frac{p(p+1)(2 p+1)}{3}-2 p$; a number of additions equal to $\frac{n^{2}}{p}+3 \frac{n}{p}+n+n p+\frac{p(p+1)(2 p+1)}{3}-3 p-2$, and a number of scalar divisions equal to $p(p+1)$. Hence, assuming again that the time expended to calculate a scalar product is equal to the time expended to calculate a division, and neglecting the time used to calculate additions, the number of floating point operations per iteration performed by the MSD-CG algorithm is given by:

$$
\frac{n^{2}}{p}+3 n+3 \frac{n}{p}+\frac{p(p+1)(2 p+1)}{3}+p(p-1)
$$

However, it is not proved that the MSD-CG algorithm converges in a finite number of iterations [13, p. 1140], and, indeed, this is not expected to occur, essentially because the linear independence of the columns of $\left[\mathbf{D}_{i}\right]_{0}^{k}$ is lost. The only available result is that the convergence rate is at least as fast as that of the steepest descent method [14, p. 1294]. Another drawback of the MSD-CG method is that communication between all the threads is required after every operation.

\section{Computational experiments}

This section reports on experimental results obtained with both randomly generated linear systems of low dimension, as well as larger dimensional matrices that arise in real applications. 
Table 3 Properties of randomly generated s.p.d. test matrices: size, condition number

\begin{tabular}{|c|c|c|}
\hline Matrix & Dimension & Condition no. \\
\hline \hline A1 & 50 & $10^{3}$ \\
\hline A2 & 50 & $10^{4}$ \\
\hline A3 & 50 & $10^{5}$ \\
\hline B1 & 100 & $10^{3}$ \\
\hline B2 & 100 & $10^{4}$ \\
\hline B3 & 100 & $10^{5}$ \\
\hline C1 & 200 & $10^{3}$ \\
\hline C2 & 200 & $10^{4}$ \\
\hline C3 & 200 & $10^{5}$ \\
\hline D1 & 300 & $10^{3}$ \\
\hline D2 & 300 & $10^{4}$ \\
\hline D3 & 300 & $10^{5}$ \\
\hline E3 & 1000 & $10^{5}$ \\
\hline
\end{tabular}

5.1 Experiments on dense randomly generated matrices of low dimension

For the experiments reported in this section, a suite of randomly generated symmetric positive definite matrices of small dimensions will be used for illustrative purposes. The dimensions of the matrices are 50, 100, 200, 300 and 1000 , respectively, and they have condition numbers of $10^{3}, 10^{4}$ and $10^{5}$. The right hand side $\mathbf{b}$ is also randomly chosen. Table 3 shows the matrix label, its dimension and condition number. In all cases, the CCG algorithm was tested from 20 different initial points per thread (i.e., 20 executions of the CCG algorithm from every initial point per thread). All these initial points are localized on a hypersphere of norm 1 centred on the known solution point, that is $\left\|\mathbf{x}_{0_{i}}-\mathbf{x}^{*}\right\|=1, \forall i \in\{1, \ldots, 20\}$. The matrices tested, as well as the right hand sides and the initial points are available on request.

The stopping criterion adopted is that at least one thread have a norm of its residue smaller than $10^{-8}$. We test the CG algorithm (one thread), the CCG algorithm with two and three threads and, for comparative purposes, the MSD-CG algorithm was also implemented.

The 20 initial points tested in the MSD-CG (as well as the CG algorithm) are the same used by the first thread in the CCG algorithm. The partition into subdomains of the vector $\mathbf{d}_{k}$ which results in the matrix $\mathbf{D}_{k}$ was made according to the criterion given in $[13$, p. 1136]. Two and three threads were tested. The stopping criterion used was $\left\|\mathbf{r}_{k}\right\|<10^{-8}$.

Table 4 reports the mean number of iterations from every initial point and the mean number of floating point operations (proportional to the time of convergence, neglecting the communication time and the time expended to calculate additions) realized in each case. 
Table 4 Mean number of iterations (it.) and mean number of floating point operations (flop) for every matrix tested. $\mathbf{b} \neq \mathbf{0}$. In the CCG algorithm flop $=$ it. $\left(n^{2}+6 n p+\frac{p(p+1)(2 p+1)}{3}+p(p-1)\right)$. Stopping criteria $\left\|\mathbf{r}_{i}\right\|<10^{-8}$ for some $i \in\{1, \ldots, p\}$. In the MSD-CG algorithm flop $=$ it. $\left(\frac{n^{2}}{p}+3 n+3 \frac{n}{p}+\frac{p(2 p+1)(p+1)}{3}+p(p-1)\right)$. Stopping criteria $\|\mathbf{r}\|<10^{-8}$. The algorithms assume that rank degeneracy may occur (so $p$ may be nonconstant). Trajectories were initiated at 20 different initial points per thread, all of them with norm one, surrounding the solution point. In the MSD-CG algorithm, each initial thread is a partition of every initial point. For each matrix, the floating point operation count in boldface occurs in the column corresponding to the algorithm that minimized this number.

\begin{tabular}{|c|c|c|c|c|c|c|c|c|c|c|}
\hline \multirow{3}{*}{ matrix } & \multirow{2}{*}{\multicolumn{2}{|c|}{$\begin{array}{c}\text { CG } \\
p=1\end{array}$}} & \multirow{2}{*}{\multicolumn{4}{|c|}{$\mathrm{CCG}$}} & \multirow{2}{*}{\multicolumn{4}{|c|}{ MSD-CG }} \\
\hline & & & & & & $=3$ & & & & 3 \\
\hline & it. & flop & it. & flop & it. & flop & it. & flop & it. & flop \\
\hline A1 & $4 \overline{48.65}$ & 136320 & 25.95 & 80750 & 17.45 & $\overline{59923}$ & 159.1 & 236580 & 160.15 & 1770930 \\
\hline A2 & 52 & 145704 & 27.35 & 85113 & 21.5 & 73831 & 405.35 & 602760 & 443.1 & 472940 \\
\hline A3 & 50.8 & 142340 & 32.1 & 97875 & 19.9 & 68337 & 216.9 & 322530 & 235.25 & 251090 \\
\hline B1 & 64.85 & 687540 & 42.25 & 507343 & 33.75 & 399400 & 158.1 & 863540 & 155.7 & 586570 \\
\hline B2 & 74.85 & 793560 & 48.7 & 546020 & 34.4 & 407090 & 263.8 & $1.4410^{6}$ & 279.85 & $1.0510^{6}$ \\
\hline B3 & 90.1 & 955240 & 90.4 & 963270 & 53.75 & 627110 & 268.55 & $1.4610^{6}$ & 300.85 & $1.1310^{6}$ \\
\hline $\mathrm{C} 1$ & 106.95 & $4.4010^{6}$ & 71.55 & $3.0310^{6}$ & 56.35 & $2.4510^{6}$ & 240.05 & $5.0210^{6}$ & 257.4 & $3.6410^{6}$ \\
\hline $\mathrm{C} 2$ & 117.5 & $4.8410^{6}$ & 81.2 & $3.4410^{6}$ & 61.85 & $2.6910^{6}$ & 402.9 & $8.4210^{6}$ & 407.9 & $5.7810^{6}$ \\
\hline $\mathrm{C} 3$ & 117.25 & $4.8310^{6}$ & 79.2 & $3.3510^{6}$ & 66.7 & $2.9010^{6}$ & 414 & $8.6510^{6}$ & 446.35 & $6.3210^{6}$ \\
\hline D1 & 89.5 & $8.2110^{6}$ & 67 & $6.2710^{6}$ & 57 & $5.4410^{6}$ & 157.75 & $7.3110^{6}$ & 155.4 & $4.8510^{6}$ \\
\hline D2 & 179.45 & $16.4710^{6}$ & 112.95 & $10.5710^{6}$ & 85.85 & $8.1910^{6}$ & 437.4 & $20.2710^{6}$ & 465.7 & $14.5410^{6}$ \\
\hline D3 & 152 & $13.9510^{6}$ & 107.85 & $10.0910^{6}$ & 86.26 & $8.2310^{6}$ & 576.55 & $26.7310^{6}$ & 607.5 & $18.9710^{6}$ \\
\hline E3 & 224.75 & $2.2610^{8}$ & 168.25 & $1.7010^{8}$ & 139.7 & $1.4210^{8}$ & 406.35 & $2.0510^{8}$ & 409.25 & $1.3810^{8}$ \\
\hline
\end{tabular}

\subsection{Experiments on dense randomly generated matrices of larger dimension}

This section reports on a suite of numerical experiments carried out on a set of random symmetric positive definite matrices of dimensions varying from 1000 to 25000 , the latter being the largest dimension that could be accommodated in the fast access RAM memory of the multicore processor. The random symmetric matrices were generated using a $\mathrm{C}$ translation of Shilon's MATLAB code [24], which produces a matrix distribution uniform over the manifold of orthogonal matrices with respect to the induced $\mathbb{R}^{n^{2}}$ Lebesgue measure. The right hand sides and initial conditions were also randomly generated, with all entries uniformly distributed on the interval $[-10,10]$. In this section, the matrices used were dense and the use of preconditioners was not investigated.

The matrices used, the right hand sides and initial conditions as well as the numerical results of the tests (omitted here for lack of space) are available on request.

In order to evaluate the performance of the algorithm proposed in this paper, a program was written in language C. The compiler used was the GNU Compiler Collection (GCC), running under Linux Ubuntu 10.0.4. For the Linear Algebra calculations, we used the Linear Algebra Package (LAPACK) and the Basic Linear Algebra Subprograms (BLAS). Finally, in order to parallelize the program, we used the Open Multi Processing (OMP) API. The processor used was an Intel Core2Quad CPU Q8200 running at $2.33 \mathrm{MHz}$ with four cores. 


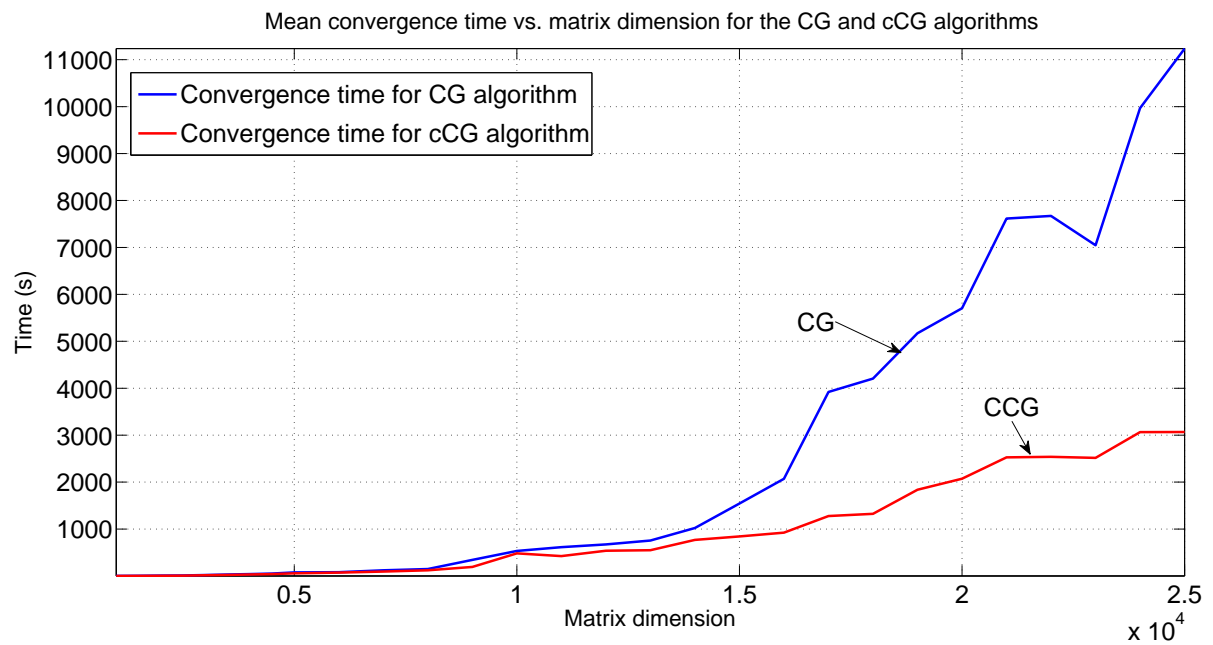

Fig. 1 Mean time to convergence for random test matrices of dimensions varying from 1000 to 25000 , condition number equal to $10^{6}$, for 3 thread CCG and standard CG algorithms.

\subsubsection{Experimental evaluation of speedup}

The results of the cooperative 3 thread CCG, in comparison with standard CG, with a tolerance of $10^{-3}$, and matrices with different sizes, but all with the same condition number of $10^{6}$, are shown in Figure 1. Multiple tests were performed, using different randomly generated initial conditions (20 different initial conditions for the small matrices and 10 for the bigger ones). Figure 1 shows the mean values computed for these tests. The iteration speedup of CCG in comparison with CG is defined as the mean number of iterations from every initial point that $\mathrm{CG}$ took to converge divided by the mean number of iterations that CCG took to converge, i.e.:

$$
\text { Iteration } \operatorname{speedup}(p)=\frac{\text { mean number of iterations } \mathrm{CG}}{\text { mean number of iterations } \mathrm{CCG}(p)}
$$

Similarly, the time speedup (classical speedup) is the ratio of the time to convergence, that is, the mean time taken by the $\mathrm{CG}$ algorithm to run the main loop until convergence divided by the mean time taken by the CCG algorithm from every initial point, i.e.:

$$
\text { Time speedup }(p)=\frac{\text { mean time of convergence CG }}{\text { mean time of convergence CCG }(p)}
$$

The experimental speedups for each dimension are shown in Figure 2. The iteration and classical speedups seem to be roughly equal up to a certain size of matrix $(n=16000)$; however, above this dimension, there is an increasing trend for both speedups. 


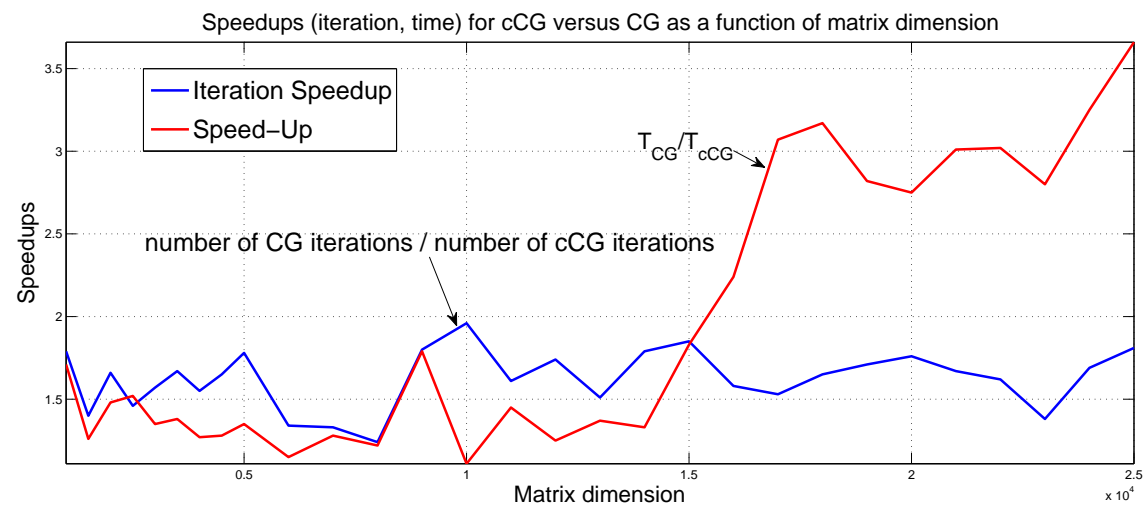

Fig. 2 Average speedups of Cooperative 3-thread CCG over classic CG for random test matrices of dimensions varying from 1000 to 25000 , condition number equal to $10^{6}$.

The numerical results obtained show that CCG, using 3 threads, leads to an improvement in comparison with the usual CG algorithm. The average iteration speedup and the classical speedup of CCG are respectively, 1.62 and 1.94, indicating that CCG converges almost twice as fast as CG for dense matrices with reasonably well-separated eigenvalues.

\subsubsection{Verifying the flop count estimates}

Figure 3 shows the mean time spent per iteration in seconds (points plotted as squares), versus matrix dimension, as well as the parabola fitted to this data, using least squares. Using the result from the last row of table 2 and multiplying it by the mean time per scalar multiplication, we obtain the parabola (dash-dotted line in Figure 3) expected in theory. In order to estimate the time per scalar multiplication, we divided the experimentally obtained mean total time spent on each iteration and divided it by the number of scalar multiplications performed in each iteration. This was done for each matrix dimension. Since the multicore threads being used for all experiments are identical, each of these divisions should generate the same value of time taken to carry out each scalar multiplication, regardless of matrix dimension. It was observed that these divisions produced a data set which has a mean value of 8.10 nanoseconds per scalar multiplication, with a standard deviation of 1.01 nanoseconds, showing that the estimate is reasonable. From equation (22), substituting $p=3$, neglecting lower order terms, and multiplying it by the estimated mean time per scalar multiplication (8.10 nanoseconds), the number of matrix multiplications per iteration, $N_{C C G}(p), p=3$, is a cubic polynomial in $n$. Thus, the logarithm of the dimension $(n)$ of the problem versus the logarithm of time needed to convergence is expected to be a straight line of slope 3 . Figure 4 shows this straight line, fitted to the data (squares) by least squares. Its slope (2.542) is fairly close to 3 , and data seems to follow a linear trend. The devia- 


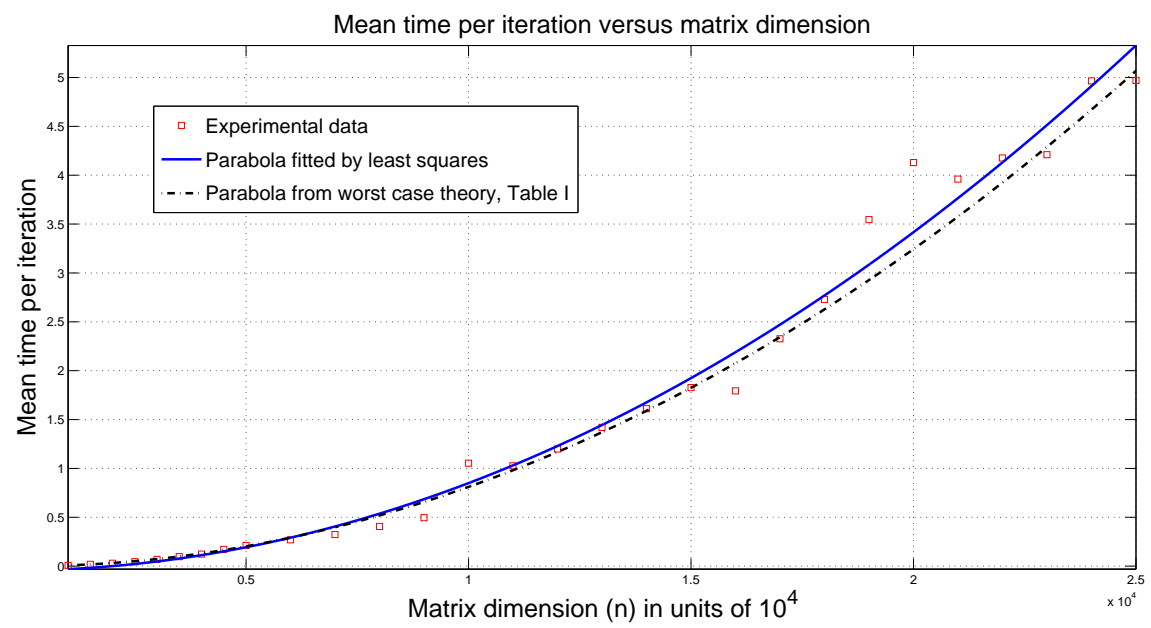

Fig. 3 Mean time per iteration versus problem dimension

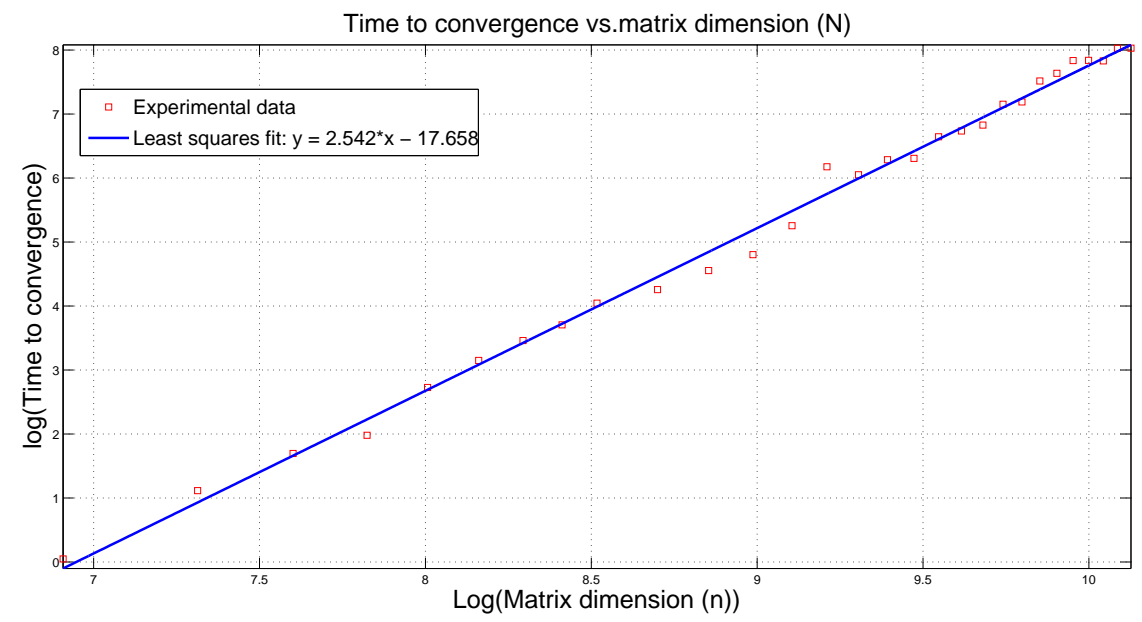

Fig. 4 Log-log plot of mean time to convergence versus problem dimension

tion of the slope from the ideal value has several probable causes, the first one being that the exact exponent of 3 is a result of a worst case analysis of CG in exact arithmetic. It is known that CG usually converges, to a reasonable tolerance, in much less than $n$ iterations [20].

Similarly, the logarithm of the number of iterations needed to convergence versus the logarithm of the dimension of the problem should also follow a linear trend. Since the number of iterations is expected to be $n / 3$, the slope of this line should be 1 . This log-log plot is shown in figure 5 , in which the 


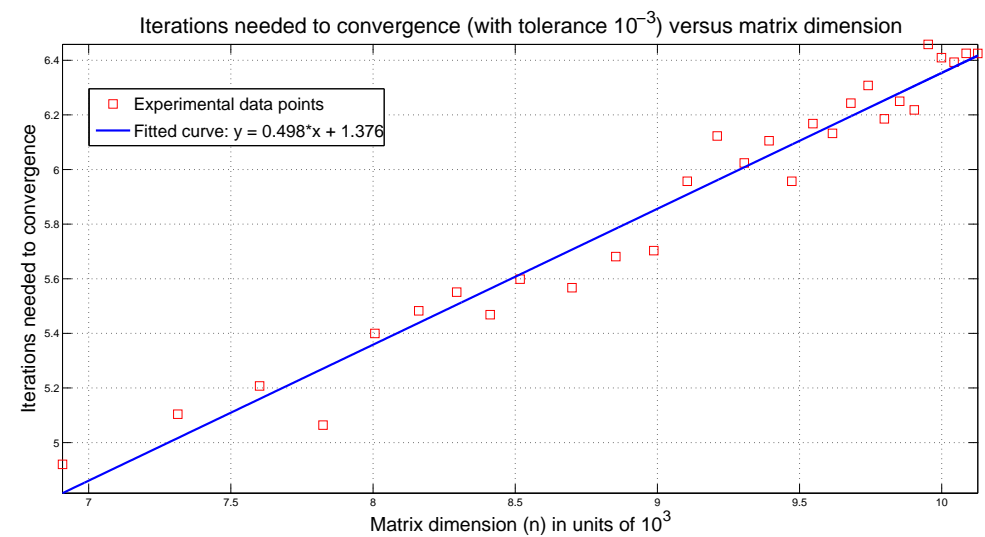

Fig. 5 Iterations needed to convergence versus problem dimension

straight line was fitted by least squares to the original data (red squares). The slope $(0.501)$ of the fitted line is smaller than 1 , but is seen to fit the data well (small residuals). The fact that both slopes are smaller than their expected values indicates that the CCG algorithm is converging faster than the worst case estimate. Another reason is that a fairly coarse tolerance of $10^{-3}$ is used, and experiments reported show that decreasing the tolerance favors the CCG algorithm even more.

\subsection{Experiments on sparse matrices arising from real applications}

In this section the results of tests carried out with a suite of sparse symmetric positive definite matrices which arise from real applications are reported. The matrices chosen were taken from [1] and their characteristics are shown in Table 5. The right hand side $\mathbf{b}$ was randomly chosen as well as the initial conditions, with all entries uniformly distributed on the interval $[-10,10]$. The tests were performed from 5 different initial points per thread. The stopping criterion was that at least one thread has a norm of its residual vector lower than $10^{-8}$.

The right hand sides used, the initial conditions as well as the numerical results of the tests are available on request.

The code for the CCG algorithm was written in language $\mathrm{C}$, with compiler GNU Compiler Collection (GCC), running under Linux Ubuntu 10.0.4. We also used the Linear Algebra Package (LAPACK) and the Basic Linear Algebra Subprograms (BLAS). Finally, in order to parallelize the program, we used the Open Multi Processing (OMP) API. The processor used was an Intel Core i7$47703.4 \mathrm{GHz}$.

The tests were performed varying the number of threads from 1 (classical $\mathrm{CG}$ ) to 8 and reporting the mean number of iterations to reach the stopping 
Table 5 Sparse symmetric positive definite matrices extracted from [1]. Dimension, number of entries different to zero and approximate condition number. The dash means that the condition number was not specified or calculated.

\begin{tabular}{|c||c|c|c|}
\hline name & dimension $n$ & nonzeros & cond. number \\
\hline \hline HB $\backslash$ bcsstk14 & 1806 & 63454 & $1.31 e 10$ \\
\hline HB $\backslash$ bcsstk18 & 11948 & 149090 & $6.486 e 11$ \\
\hline Lourakis $\backslash$ bundle1 & 10581 & 770811 & $1.3306 e 4$ \\
\hline TKK $\backslash$ cbuckle & 13681 & 676515 & $8.0476 e 7$ \\
\hline JGD-Trefethen $\backslash$ Trefethen-20000b & 19999 & 554435 & - \\
\hline JGD-Trefethen $\backslash$ Trefethen-20000 & 20000 & 554466 & - \\
\hline MathWorks $\backslash$ Kuu & 7102 & 340200 & $3.2553 e 4$ \\
\hline Pothen $\backslash$ bodyy4 & 17546 & 121550 & $1.016 e 3$ \\
\hline Pothen $\backslash$ bodyy5 & 18589 & 128853 & $9.9769 e 3$ \\
\hline Pothen $\backslash$ bodyy6 & 19366 & 134208 & $9.7989 e 4$ \\
\hline UTEP $\backslash$ Dubcova1 & 16129 & 253009 & $2.6247 e 3$ \\
\hline HB $\backslash$ gr-30-30 & 900 & 7744 & 377.23 \\
\hline
\end{tabular}

criterion and the mean time of convergence of the 5 executions performed from each one of the different initial points.

For illustrative purposes, Figures 6,7 and 8 show the speedups for a number of threads varying from 1 to 8 with respect to the classical CG algorithm for the matrices bcsstk18, cbuckle and gr-30-30, respectively.

Note that if the CCG algorithm and the CG algorithm converge in the worst case, the numbers of iterations to converge are $\left\lceil\frac{n}{p}\right\rceil$ and $n$, respectively, and hence for $n \gg p$ the expected iteration speedup is equal to $p$ (a straight line of slope 1). Similarly, the expected time speedup in the worst case is given by the time to convergence of the CG algorithm, which is proportional to $N_{C G}$ given by (23) divided by the time to convergence taken by the CCG algorithm, which is proportional to $N_{C C G}(p)$ given by (22); for large $n$ this expected time speedup in the worst case is also approximately equal to $p$.

$$
\text { Expected worst case time speedup }=\frac{n^{3}+6 n^{2}+2 n}{\frac{n^{3}}{p}+6 n^{2}+\frac{2}{3} n p^{2}+2 n p-\frac{2}{3} n}
$$

Figure 9 shows the iteration speedup and the time speedup for each matrix reported in Table 5 and for each thread tested from 1 to 8. Figure 9 shows that for all the matrices tested, the greater the number of threads, the greater the speedups, exactly as expected when a small number of threads is used and as it happens in the worst case. For some matrices, an increase in the number of threads does not increase the speedup significantly, as observed with the matrices bodyy4, bodyy 5 and bodyy 6 , whereas with other matrices the increase of the speedup with the number of threads is much greater. Further research is needed to explain these results and correlate them to, for example, the eigenvalue distribution of the matrices.

Figure 10 shows the average iteration and average time speedups for the 12 matrices reported in Table 5. Figure 10 confirms the trend of speedup increasing with the number of threads. 


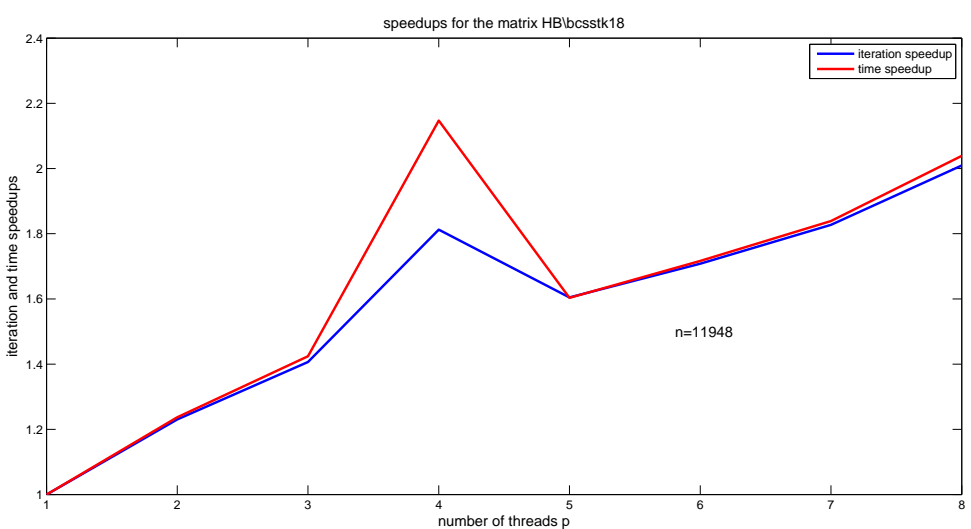

Fig. 6 Iteration speedup and time speedup for the matrix $H B \backslash$ bcsstk18 vs. number of threads $p$.

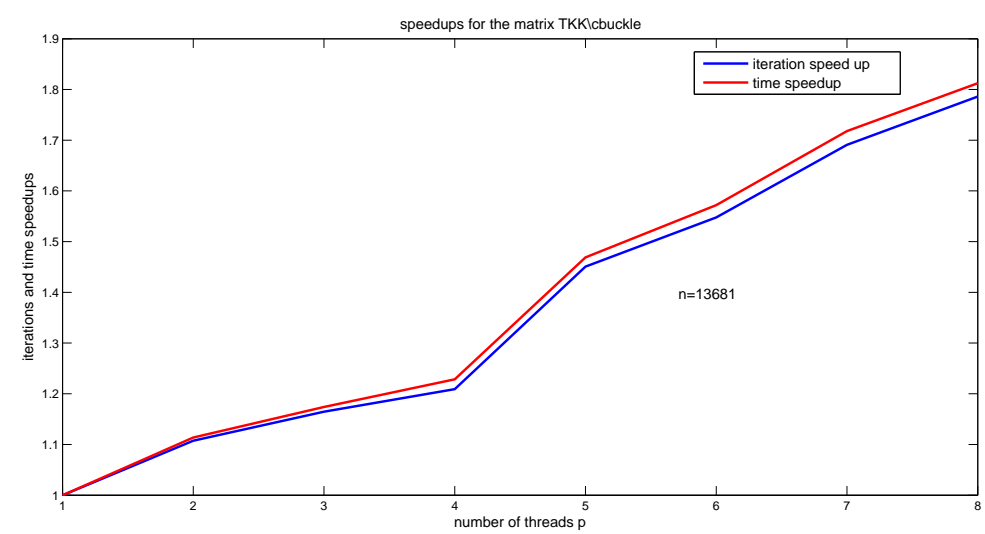

Fig. 7 Iteration speedup and time speedup for the matrix TKK\cbuckle vs. number of threads $p$.

\section{Conclusions}

This paper revisited some existing block and multiparameter CG algorithms in the new context of multi-thread computing, proposing a cooperative conjugate gradient (CCG) method for linear systems with symmetric positive definite coefficient matrices. This CCG method permits efficient implementation on a multicore computer and experimental results bear out the main theoretical properties, namely, that speedups close to the theoretical value of $p$, when a $p$-core computer is used, are possible, when the matrix dimension is suitably large. 


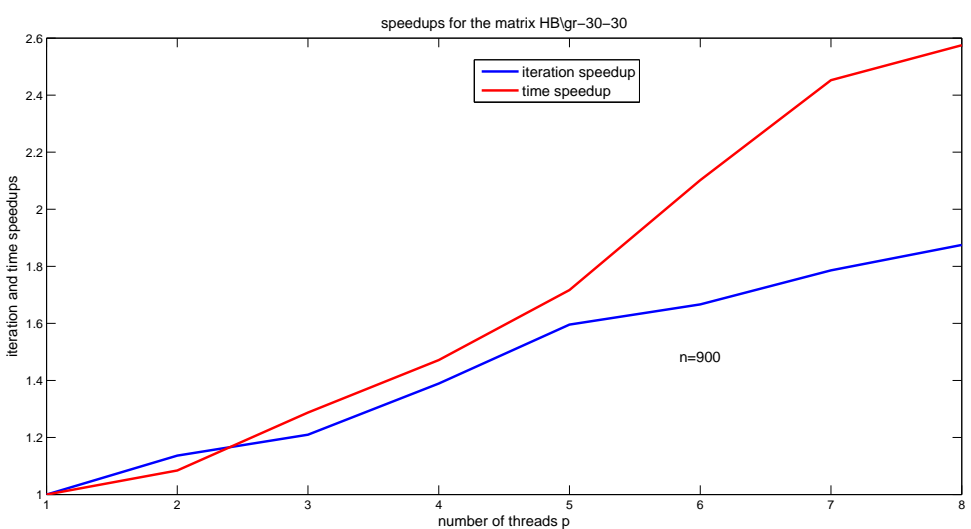

Fig. 8 Iteration speedup and time speedup for the matrix $\mathrm{HB} \backslash \mathrm{gr}-30-30$ vs. number of threads $p$.
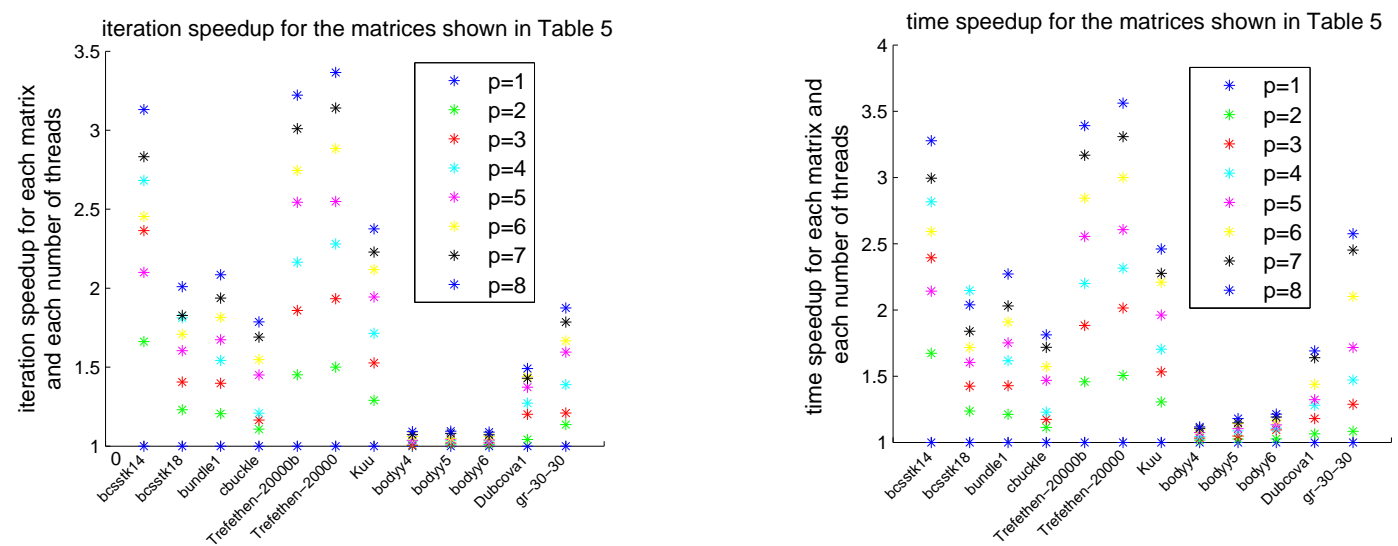

Fig. 9 Iteration speedup (left) and time speedup (right) for each matrix reported in Table 5 and for each number of threads used by the CCG algorithm.

The experimental results were carried out with dense randomly generated matrices as well as with matrices arising from real applications, which are typically sparse and sometimes ill-conditioned. In all the cases, the increase of the speedups with the number of threads was observed, although the results are less significant for some matrices than for others, which is a topic requiring further investigation.

The comparison with the other multi-thread block CG method presented in the literature, the MSD-CG $[13,14]$ showed that the CCG algorithm converges faster than the MSD-CG (with the same number of threads), in almost all 


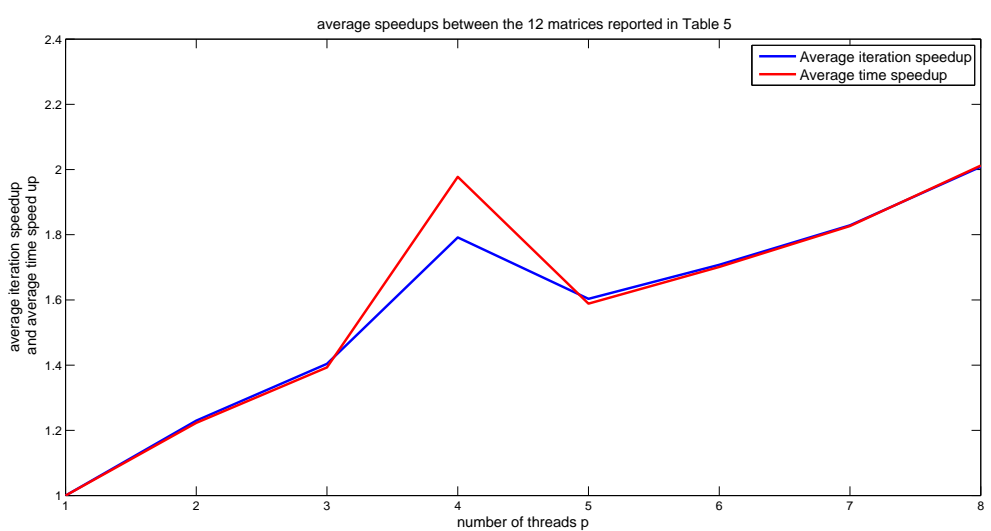

Fig. 10 Average iteration speedup and time speedup for the 12 matrices reported in Table 5 vs. number of threads $p$.

cases. The tests with large matrices, either dense and randomly generated or sparse arising from real applications, show that the CCG algorithm is faster than the classic CG and that the speedup increases with the number of threads.

The use of processors with a larger number of threads should also permit further exploration of the notable theoretical result of Corollary 2 that, in the asymptotic limit, as $n$ becomes large, implying that the optimal number of threads $p^{*}$ also increases according to (25a), solution of $\mathbf{A x}=\mathbf{b}$ is possible by the method proposed here with a worst-case cost of $O\left(n^{2+\frac{1}{3}}\right)$ floating point operations.

\section{References}

1. Sparse matrix collection. Available at http://www.cise.ufl.edu/research/sparse/matrices/ (2009)

2. Abkowicz, A., Brezinski, C.: Acceleration properties of the hybrid procedure for solving linear systems. Applicationes Mathematicae 4(23), 417-432 (1996)

3. Bantegnies, F., Brezinski, C.: The multiparameter conjugate gradient algorithm. Tech. Report 429, Laboratoire d'Analyse Numérique et d'Optimisation. Université des Sciences et Technologies de Lille, Lille, France (2001)

4. Bhaya, A., Bliman, P.A., Niedu, G., Pazos, F.: A cooperative conjugate gradient method for linear systems permitting multithread implementation of low complexity. ArXiv eprints (2012)

5. Bhaya, A., Bliman, P.A., Niedu, G., Pazos, F.: A cooperative conjugate gradient method for linear systems permitting multithread implementation of low complexity. In: Proc. of the 51st IEEE Conference on Decision and Control. Maui, Hawaii, USA (2012)

6. Bhaya, A., Bliman, P.A., Pazos, F.: Cooperative parallel asynchronous computation of the solution of symmetric linear systems. In: Proc. of the 49th IEEE Conference on Decision and Control. Atlanta, USA (2010)

7. Bouyouli, R., Meurant, G., Smoch, L., Sadok, H.: New results on the convergence of the conjugate gradient method. Numerical Linear Algebra with Applications pp. 1-12 (2008) 
8. Brezinski, C.: Multiparameter descent methods. Linear Algebra and its Applications 296, 113-141 (1999)

9. Brezinski, C., Chehab, J.P.: Nonlinear hybrid procedures and fixed point iterations. Numer. Funct. Anal. Optimization 19, 465-487 (1998)

10. Brezinski, C., Redivo-Zaglia, M.: Hybrid procedures for solving linear systems. Numerische Mathematik (67), 1-19 (1994)

11. Bridson, R., Greif, C.: A multipreconditioned conjugate gradient algorithm. SIAM Journal Matrix Anal. Appl. 27(4), 1056-1068 (2006)

12. Greenbaum, A.: Iterative methods for solving linear systems. SIAM, Philadelphia (1997)

13. Gu, T., Liu, X., Mo, Z., Chi, X.: Multiple search direction conjugate gradient method 1: Methods and their propositions. International Journal of Computer Mathematics 81(9), 1133-1143 (2004)

14. Gu, T., Liu, X., Mo, Z., Chi, X.: Multiple search direction conjugate gradient method 2: Theory and numerical experiments. International Journal of Computer Mathematics 81(10), 1289-1307 (2004)

15. Güler, O.: Foundations of optimization. Graduate texts in mathematics. Springer, New York (2010)

16. Gutknecht, M.H.: Block Krylov space methods for linear systems with multiple righthand sides: an introduction. In: I.D. A.H. Siddiqi, O. Christensen (eds.) Modern Mathematical Models, Methods and Algorithms for Real World Systems, pp. 420-447. Anamaya Publishers, New Delhi, India (2007)

17. Hestenes, M.R., Stiefel, E.: Methods of conjugate gradients for solving linear systems. Journal of Research of the National Bureau of Standards 49, 409-436 (1952)

18. Kumar, V., Leonard, N., Morse, A.S. (eds.): 2003 Block Island Workshop on Cooperative Control, Lecture Notes in Control and Information Sciences, vol. 309. Springer (2005)

19. Luenberger, D.G., Ye, Y.: Linear and nonlinear programming, 3 edn. Springer, New York (2008)

20. Meurant, G., Strakoš, Z.: The Lanczos and conjugate gradient algorithms in finite precision arithmetic. Acta Numerica 15, 471-542 (2006)

21. Murray, R.M.: Recent research in cooperative control of multi-vehicle systems. J. Guidance, Control and Dynamics 129(5), 571-583 (2007)

22. Nedic, A., Ozdaglar, A.: Convex Optimization in Signal Processing and Communications, chap. Cooperative Distributed Multi-agent Optimization, pp. 340-386. Cambridge University Press (2010)

23. O'Leary, D.P.: The block conjugate gradient algorithm and related methods. Linear Algebra with Applications (29), 293-322 (1980)

24. Shilon, O.: RandOrthMat.m: MATLAB code to generate a random $n \times n$ orthogonal real matrix (2006). Http://www.mathworks.com/matlabcentral/fileexchange/authors/23951

25. Strang, G.: Linear algebra and its applications. Harcourt Brace Jovanovich, San Diego, California (1988)

\section{Appendix: Proofs of results in sections 3.1 and 3.2}

Proof of theorem 4.

For all $i \in\{1, \ldots, p\}$, denoting

$$
h_{i}\left(\boldsymbol{\gamma}_{i_{0}}, \boldsymbol{\gamma}_{i_{1}}, \ldots, \boldsymbol{\gamma}_{i_{k-1}}\right):=f\left(\mathbf{x}_{i_{0}}+\mathbf{D}_{0} \boldsymbol{\gamma}_{i_{0}}^{\top}+\mathbf{D}_{1} \boldsymbol{\gamma}_{i_{1}}^{\top}+\cdots+\mathbf{D}_{k-1} \boldsymbol{\gamma}_{i_{k-1}}^{\top}\right) \in \mathbb{R}
$$

where $\boldsymbol{\gamma}_{i_{\ell}} \in \mathbb{R}^{1 \times p}, \ell \in\{0, \ldots, k-1\}$ are row vectors; the coefficients $\boldsymbol{\gamma}_{i_{\ell}}$ that minimize the scalar function $f(\mathbf{x})$ on the affine set $\mathbf{x}_{i_{0}}+\operatorname{span}\left[\mathbf{D}_{j}\right]_{0}^{k-1}$ are given by:

$$
\begin{aligned}
\forall \ell<k: \quad \frac{\partial h_{i}}{\partial \gamma_{i_{\ell}}}{ }^{\top} & =\nabla^{\top} f\left(\mathbf{x}_{i_{0}}+\mathbf{D}_{0} \boldsymbol{\gamma}_{i_{0}}^{\top}+\mathbf{D}_{1} \boldsymbol{\gamma}_{i_{1}}^{\top}+\cdots+\mathbf{D}_{k-1} \boldsymbol{\gamma}_{i_{k-1}}^{\top}\right) \mathbf{D}_{\ell} \\
& =\left(\mathbf{r}_{i_{0}}+\mathbf{A} \mathbf{D}_{0} \boldsymbol{\gamma}_{i_{0}}^{\top}+\mathbf{A D}_{1} \boldsymbol{\gamma}_{i_{1}}^{\top}+\cdots+\mathbf{A} \mathbf{D}_{k-1} \boldsymbol{\gamma}_{i_{k-1}}^{\top}\right)^{\top} \mathbf{D}_{\ell} \\
& =\mathbf{r}_{i_{0}}^{\top} \mathbf{D}_{\ell}+\boldsymbol{\gamma}_{i_{\ell}} \mathbf{D}_{\ell}^{\top} \mathbf{A D}_{\ell}=\mathbf{0}
\end{aligned}
$$


which implies that $\gamma_{i_{\ell}}=-\mathbf{r}_{i_{0}}^{\top} \mathbf{D}_{\ell}\left(\mathbf{D}_{\ell}^{\top} \mathbf{A D}_{\ell}\right)^{-1} \in \mathbb{R}^{1 \times p}$; and considering all the row vectors $i \in\{1, \ldots, p\}$ :

$$
\gamma_{\ell}=-\mathbf{R}_{0}^{\top} \mathbf{D}_{\ell}\left(\mathbf{D}_{\ell}^{\top} \mathbf{A} \mathbf{D}_{\ell}\right)^{-1} \in \mathbb{R}^{p \times p}
$$

For all $\mathbf{x} \in \mathbf{x}_{i_{0}}+\operatorname{span}\left[\mathbf{D}_{j}\right]_{0}^{\ell-1}: \quad \mathbf{x}=\mathbf{x}_{i_{0}}+\mathbf{D}_{0} \boldsymbol{\delta}_{0}^{\top}+\cdots+\mathbf{D}_{\ell-1} \boldsymbol{\delta}_{\ell-1}^{\top}$, where $\boldsymbol{\delta}_{j} \in$ $\mathbb{R}^{1 \times p}, \forall j \in\{0, \ldots, \ell-1\}$. Thus:

$$
\nabla f(\mathbf{x})=\mathbf{r}_{i_{0}}+\mathbf{A D}_{0} \boldsymbol{\delta}_{0}^{\top}+\cdots+\mathbf{A} \mathbf{D}_{\ell-1} \boldsymbol{\delta}_{\ell-1}^{\top}, \quad \text { which implies } \quad \nabla^{\top} f(\mathbf{x}) \mathbf{D}_{\ell}=\mathbf{r}_{i_{0}}^{\top} \mathbf{D}_{\ell}
$$

and this is valid for all $\mathbf{x} \in \mathbf{x}_{i_{0}}+\operatorname{span}\left[\mathbf{D}_{j}\right]_{0}^{\ell-1}$, hence it is valid for $\mathbf{x}_{i_{\ell}}=\mathbf{x}_{i_{0}}+\mathbf{D}_{0} \boldsymbol{\alpha}_{i_{0}}^{\top}+$ $\cdots+\mathbf{D}_{\ell-1} \boldsymbol{\alpha}_{i_{\ell-1}}^{\top}$, where $\boldsymbol{\alpha}_{i_{j}}^{\top}$ is the $i^{\text {th }}$ column of the $\boldsymbol{\alpha}_{j}^{\top}$ matrix (12) for all $j \in\{0, \ldots, \ell-1\}$.

Therefore, $\nabla^{\top} f\left(\mathbf{x}_{i_{\ell}}\right) \mathbf{D}_{\ell}=\mathbf{r}_{i_{\ell}}^{\top} \mathbf{D}_{\ell}=\mathbf{r}_{i_{0}}^{\top} \mathbf{D}_{\ell}$, and considering all the row vectors $\mathbf{r}_{i_{\ell}}^{\top}, i \in$ $\{1, \ldots, p\}$ :

$$
\mathbf{R}_{\ell}^{\top} \mathbf{D}_{\ell}=\mathbf{R}_{0}^{\top} \mathbf{D}_{\ell}
$$

and substituting in (28):

$$
\gamma_{\ell}=-\mathbf{R}_{\ell}^{\top} \mathbf{D}_{\ell}\left(\mathbf{D}_{\ell}^{\top} \mathbf{A} \mathbf{D}_{\ell}\right)^{-1}=\boldsymbol{\alpha}_{\ell} \quad \forall \ell<k
$$

which proves that the step size (12) minimizes $f\left(\mathbf{x}_{i_{k}}\right)$ on the affine set $\mathbf{x}_{i_{0}}+\operatorname{span}\left[\mathbf{D}_{j}\right]_{0}^{k-1}$ for all $i \in\{1, \ldots, p\}$.

Note also that, by (10) $\mathbf{x}_{i_{k}}=\mathbf{x}_{i_{0}}+\mathbf{D}_{0} \boldsymbol{\alpha}_{i_{0}}^{\top}+\mathbf{D}_{1} \boldsymbol{\alpha}_{i_{1}}^{\top}+\cdots+\mathbf{D}_{k-1} \boldsymbol{\alpha}_{i_{k-1}}^{\top}$, hence, for all $\ell<k$ :

$$
\begin{aligned}
& \nabla^{\top} f\left(\mathbf{x}_{i_{k}}\right) \mathbf{D}_{\ell}=\nabla^{\top} f\left(\mathbf{x}_{i_{0}}+\mathbf{D}_{0} \boldsymbol{\alpha}_{i_{0}}^{\top}+\mathbf{D}_{1} \boldsymbol{\alpha}_{i_{1}}^{\top}+\cdots+\mathbf{D}_{k-1} \boldsymbol{\alpha}_{i_{k-1}}^{\top}\right) \mathbf{D}_{\ell}= \\
& \left(\mathbf{r}_{i_{0}}+\mathbf{A D}_{0} \boldsymbol{\alpha}_{i_{0}}^{\top}+\mathbf{A D}_{1} \boldsymbol{\alpha}_{i_{1}}^{\top}+\cdots+\mathbf{A D}_{k-1} \boldsymbol{\alpha}_{i_{k-1}}^{\top}\right)^{\top} \mathbf{D}_{\ell}=\mathbf{r}_{i_{k}}^{\top} \mathbf{D}_{\ell}=\mathbf{0}
\end{aligned}
$$

and considering all the row vectors $i \in\{1, \ldots, p\}$ :

$$
\mathbf{R}_{k}^{\top} \mathbf{D}_{\ell}=0 \quad \forall \ell<k
$$

Proof of lemma 3.

By theorem 4, if $\mathbf{R}_{k}$ is orthogonal to span $\left[\mathbf{D}_{i}\right]_{0}^{k-1}$, then this is orthogonal to span $\left[\mathbf{R}_{i}\right]_{0}^{k-1}$, which means that for all $j<k$ : $\mathbf{R}_{k}^{\top} \mathbf{R}_{j}=0$.

By (10): $\forall j<k: \mathbf{X}_{j+1}=\mathbf{X}_{j}+\mathbf{D}_{j} \boldsymbol{\alpha}_{j}^{\top}$ hence $\quad \mathbf{D}_{j} \boldsymbol{\alpha}_{j}^{\top}=\mathbf{X}_{j+1}-\mathbf{X}_{j}$

which implies $\mathbf{R}_{k}^{\top} \mathbf{A} \mathbf{D}_{j} \boldsymbol{\alpha}_{j}^{\top}=\mathbf{R}_{k}^{\top}\left(\mathbf{R}_{j+1}-\mathbf{R}_{j}\right)$

Supposing rank $\mathbf{D}_{j}=p, \boldsymbol{\alpha}_{j}$ is non singular, thus $\forall j<k-1: \quad \mathbf{R}_{k}^{\top} \mathbf{A D}_{j}=0$

for $j=k-1: \quad \mathbf{R}_{k}^{\top} \mathbf{A D}_{k-1} \alpha_{k-1}^{\top}=\mathbf{R}_{k}^{\top} \mathbf{R}_{k} \neq 0 \Rightarrow \boldsymbol{\alpha}_{k-1}^{\top}=\left(\mathbf{R}_{k}^{\top} \mathbf{A D}_{k-1}\right)^{-1} \mathbf{R}_{k}^{\top} \mathbf{R}_{k}$

Using this result in (14):

$$
\begin{aligned}
\mathbf{D}_{k+1} & =\mathbf{R}_{k+1}-\sum_{j=0}^{k} \mathbf{D}_{j}\left(\mathbf{D}_{j}^{\top} \mathbf{A} \mathbf{D}_{j}\right)^{-1} \mathbf{D}_{j}^{\top} \mathbf{A} \mathbf{R}_{k+1} \\
& =\mathbf{R}_{k+1}-\mathbf{D}_{k}\left(\mathbf{D}_{k}^{\top} \mathbf{A} \mathbf{D}_{k}\right)^{-1} \mathbf{D}_{k}^{\top} \mathbf{A} \mathbf{R}_{k+1} \\
& =\mathbf{R}_{k+1}-\mathbf{D}_{k}\left(\mathbf{R}_{k+1}^{\top} \mathbf{A D}_{k}\left(\mathbf{D}_{k}^{\top} \mathbf{A} \mathbf{D}_{k}\right)^{-1}\right)^{\top}=\mathbf{R}_{k+1}+\mathbf{D}_{k} \boldsymbol{\beta}_{k}^{\top}
\end{aligned}
$$

which coincides with (11) with step size (13), thus proving that the matrices generated by this method are also conjugate.

Proof of Lemma 6.

By induction. Since the columns of $\mathbf{D}_{0}$ are linearly independent by hypotheses, supposing rank $\left[\mathbf{D}_{i}\right]_{0}^{k-1}=p k$, it is enough to prove that the columns of $\left[\mathbf{D}_{i}\right]_{0}^{k}$ also are linearly independent if $p(k+1) \leq n$.

By equation (11): $\mathbf{D}_{k+1}=\mathbf{R}_{k+1}+\mathbf{D}_{k} \boldsymbol{\beta}_{k}^{\top}$.

Evidently, $\left\{\mathbf{d}_{i_{k}}\right\}_{1}^{p} \subset \operatorname{span}\left[\mathbf{D}_{i}\right]_{0}^{k}$. Using the proof of the lemma $3,\left\{\mathbf{r}_{i_{k+1}}\right\}_{1}^{p} \subset \operatorname{span}\left[\mathbf{A}^{i} \mathbf{R}_{0}\right]_{0}^{k+1}=$ span $\left[\mathbf{D}_{i}\right]_{0}^{k+1}$ and $\left\{\mathbf{r}_{i_{k+1}}\right\}_{1}^{p} \not \subset$ span $\left[\mathbf{A}^{i} \mathbf{R}_{0}\right]_{i}^{k}=$ span $\left[\mathbf{D}_{i}\right]_{0}^{k}$ because $\mathbf{R}_{k+1}$ is orthogonal to 
span $\left[\mathbf{D}_{i}\right]_{0}^{k}$. Hence, the columns of $\mathbf{R}_{k+1}$ can be expressed neither as a linear combination of $\left[\mathbf{D}_{0} \cdots \mathbf{D}_{k}\right]$, and, from (11), nor as a linear combination of the columns of $\mathbf{D}_{k+1}$, which means that the columns of $\left[\mathbf{D}_{0} \cdots \mathbf{D}_{k} \mathbf{D}_{k+1}\right]=\left[\mathbf{D}_{i}\right]_{0}^{k+1}$ are linearly independent.

Note that this linear independence persists until an iteration $k$ such that $p(k+1) \geq n$, and in this case $\left[\mathbf{D}_{i}\right]_{0}^{k} \in \mathbb{R}^{n \times p(k+1)}$ and rank $\left[\mathbf{D}_{i}\right]_{0}^{k}=n \leq p(k+1)$.

\section{Proof of theorem 5.}

By lemma $6\left[\mathbf{D}_{0} \cdots \mathbf{D}_{k^{*}-1}\right] \in \mathbb{R}^{n \times p k^{*}}$, and $\operatorname{rank}\left[\mathbf{D}_{0} \cdots \mathbf{D}_{k^{*}-1}\right]=n=p k^{*}$. Hence, every vector $\mathbf{x}^{*}-\mathbf{x}_{i_{0}}, \forall i \in\{1, \ldots, p\}$ can be expressed as a linear combination of a base of the subspace span $\left[\mathbf{D}_{i}\right]_{0}^{k^{*}-1}$ :

$$
\mathbf{x}^{*}-\mathbf{x}_{i_{0}}=\mathbf{D}_{0} \boldsymbol{\gamma}_{i_{0}}^{\top}+\mathbf{D}_{1} \boldsymbol{\gamma}_{i_{1}}^{\top}+\cdots+\mathbf{D}_{k^{*}-2} \boldsymbol{\gamma}_{i_{k^{*}-2}}^{\top}+\mathbf{D}_{k^{*}-1} \boldsymbol{\gamma}_{i_{k^{*}-1}}^{\top}
$$

where $\boldsymbol{\gamma}_{i_{k}} \in \mathbb{R}^{1 \times p}, k \in\left\{0, \ldots, k^{*}-1\right\}$ are the coefficients of the linear combination of $\mathbf{x}^{*}-\mathbf{x}_{i_{0}}$.

Thus, for all $k \in\left\{0, \ldots, k^{*}-1\right\}, i \in\{1, \ldots, p\}$ :

$$
\mathbf{D}_{k}^{\top} \mathbf{A}\left(\mathbf{x}^{*}-\mathbf{x}_{i_{0}}\right)=\mathbf{D}_{k}^{\top} \mathbf{A} \mathbf{D}_{k} \boldsymbol{\gamma}_{i_{k}}^{\top} \in \mathbb{R}^{p} \quad \Rightarrow \quad \gamma_{i_{k}}^{\top}=\left(\mathbf{D}_{k}^{\top} \mathbf{A} \mathbf{D}_{k}\right)^{-1} \mathbf{D}_{k}^{\top} \mathbf{A}\left(\mathbf{x}^{*}-\mathbf{x}_{i_{0}}\right)
$$

Following the sequence (10), from $\mathbf{x}_{i_{0}}$ to $\mathbf{x}_{i_{k}}$ for all $i \in\{1, \ldots, p\}$ :

$$
\begin{aligned}
& \mathbf{x}_{i_{k}}-\mathbf{x}_{i_{0}}=\mathbf{D}_{0} \boldsymbol{\alpha}_{i_{0}}^{\top}+\mathbf{D}_{1} \boldsymbol{\alpha}_{i_{1}}^{\top}+\cdots+\mathbf{D}_{k-1} \boldsymbol{\alpha}_{i_{k-1}}^{\top} \\
& \Rightarrow \mathbf{D}_{k}^{\top} \mathbf{A}\left(\mathbf{x}_{i_{k}}-\mathbf{x}_{i_{0}}\right)=0 \\
& \Rightarrow \mathbf{D}_{k}^{\top} \mathbf{A} \mathbf{x}_{i_{k}}=\mathbf{D}_{k}^{\top} \mathbf{A} \mathbf{x}_{i_{0}}
\end{aligned}
$$

where lemma 3 was used and $\boldsymbol{\alpha}_{i_{j}}$ is the $i^{\text {th }}$ row of the $\boldsymbol{\alpha}_{j}$ matrix calculated as in (12). Substituting in the former equation:

$$
\boldsymbol{\gamma}_{i_{k}}^{\top}=\left(\mathbf{D}_{k}^{\top} \mathbf{A} \mathbf{D}_{k}\right)^{-1} \mathbf{D}_{k}^{\top} \mathbf{A}\left(\mathbf{x}^{*}-\mathbf{x}_{i_{k}}\right)=-\left(\mathbf{D}_{k}^{\top} \mathbf{A} \mathbf{D}_{k}\right)^{-1} \mathbf{D}_{k}^{\top} \mathbf{r}_{i_{k}}
$$

and considering all the $p$ rows:

$$
\Gamma_{k}^{\top}:=\left[\boldsymbol{\gamma}_{i_{k}}^{\top}\right]_{1}^{p}=-\left(\mathbf{D}_{k}^{\top} \mathbf{A D}_{k}\right)^{-1} \mathbf{D}_{k}^{\top} \mathbf{R}_{k}
$$

which coincides with (12). Hence, the coefficients of the linear combination (32) are the step sizes $\boldsymbol{\alpha}_{k}$, and the sequence

$$
\mathbf{x}_{i_{k^{*}}}=\mathbf{x}_{i_{0}}+\mathbf{D}_{0} \boldsymbol{\alpha}_{i_{0}}^{\top}+\cdots+\mathbf{D}_{k^{*}-1} \boldsymbol{\alpha}_{i_{k^{*}-1}}^{\top}
$$

for all $i \in\{1, \ldots, p\}$ is equal to $\mathbf{x}^{*}$, thus proving that all the $p$ threads converge in $k^{*}$ iterations.

Proof of lemma $\%$.

By lemma 6 , for all $k>0$ until convergence, if $\mathbf{D}_{k} \neq 0$, then $\left\{\mathbf{d}_{i_{k}}\right\}_{1}^{p_{k}} \not \subset$ span $\left[\mathbf{D}_{i}\right]_{0}^{k-1}$ and rank $\left[\mathbf{d}_{i_{k}}\right]_{1}^{p_{k}}=p_{k} \geq 1$. Therefore, the matrix $\left[\mathbf{D}_{0} \cdots \mathbf{D}_{k^{*}-1}\right]$, where $k^{*}$ is chosen such that min rank $\left[\mathbf{D}_{0} \cdots \mathbf{D}_{k^{*}-1}\right] \geq n$, has a finite number of columns.

Finally we can choose $p_{k^{*}-1}=n-\sum_{k=0}^{k^{*}-2} p_{k}$, that is, we eliminate columns of $\mathbf{D}_{k^{*}-1}$ in such a way to have a number of columns enough to complete $n$ linearly independent columns, i.e. $\operatorname{rank}\left[\mathbf{D}_{i}\right]_{0}^{k^{*}-1}=\operatorname{rank} \mathbf{D}_{0}+\cdots+\operatorname{rank} \mathbf{D}_{k^{*}-1}=p_{0}+\cdots+p_{k^{*}-1}=n$.

Note that in the best case $p_{k}=p, \forall k \in\left\{0, \ldots, k^{*}-1\right\}$, which implies that $k^{*}=\left\lceil\frac{n}{p}\right\rceil$. In the general case $\left\lceil\frac{n}{p}\right\rceil \leq k^{*} \leq n-p+1$. 